\title{
Analysis of the domain mapping method for elliptic diffusion problems on random domains
}

\author{
H. Harbrecht · M. Peters · M. Siebenmorgen
}

the date of receipt and acceptance should be inserted later

\begin{abstract}
In this article, we provide a rigorous analysis of the solution to elliptic diffusion problems on random domains. In particular, based on the decay of the Karhunen-Loève expansion of the domain perturbation field, we establish decay rates for the derivatives of the random solution that are independent of the stochastic dimension. For the implementation of a related approximation scheme, like quasi-Monte Carlo quadrature, stochastic collocation, etc., we propose parametric finite elements to compute the solution of the diffusion problem on each individual realization of the domain generated by the perturbation field. This simplifies the implementation and yields a non-intrusive approach. Having this machinery at hand, we can easily transfer it to stochastic interface problems. The theoretical findings are complemented by numerical examples for both, stochastic interface problems and boundary value problems on random domains.
\end{abstract}

\section{Introduction}

Many problems in science and engineering lead to boundary value problems for an unknown function. In general, the numerical simulation is well understood provided that the input parameters are given exactly. Often, however, the input parameters are not known exactly. Especially, the treatment of uncertainties in the computational domain has become of growing interest, see e.g. $[5,18,33,36]$. In this article, we consider the elliptic diffusion equation

$$
-\operatorname{div}(\alpha \nabla u(\omega))=f \text { in } D(\omega), \quad u(\omega)=0 \text { on } \partial D(\omega)
$$

as a model problem where the underlying domain $D \subset \mathbb{R}^{d}$ or respectively its boundary $\partial D$ are random. For example, one might think of tolerances in the shape of

This research has been supported by the Swiss National Science Foundation (SNSF) through the project "Rapid Solution of Boundary Value Problems on Stochastic Domains".

Departement Mathematik und Informatik, Universität Basel, Spiegelgasse 1, 4051 Basel, Switzerland

E-mail: \{helmut.harbrecht,michael.peters,markus.siebenmorgen\}@unibas.ch 
products fabricated by line production or shapes which stem from inverse problems, like e.g. tomography. Besides the fictitious domain approach considered in [5], one might essentially distinguish two approaches: the perturbation method and the domain mapping method.

The perturbation method starts with a prescribed perturbation field

$$
\mathbf{V}(\omega): \partial D_{\text {ref }} \rightarrow \mathbb{R}^{d}
$$

at the boundary $\partial D_{\text {ref }}$ of a reference configuration and uses a shape Taylor expansion with respect to this perturbation field to represent the solution to $(1)$, cf. $[14,18]$. Whereas, the domain mapping method requires that the perturbation field is also known in the interior of the domain $D_{\text {ref }}$, i.e.

$$
\mathbf{V}(\omega): \overline{D_{\text {ref }}} \rightarrow \mathbb{R}^{d}
$$

Then, the problem may be transformed to the fixed reference domain $D_{\text {ref }}$. This yields a partial differential equation with correlated random diffusion matrix and right hand side, cf. $[6,26,33,36]$.

The major drawback of the perturbation method is that it is only feasible for relatively small perturbations. Thus, in order to treat larger perturbations, the domain mapping method is the method of choice. Nevertheless, it might in practice be much easier to obtain measurements from the outside of a workpiece to estimate the perturbation field $\mathbf{V}(\omega)$ rather than from its interior. If no information of the vector field inside the domain is available, it has to be extended appropriately, e.g. by the Laplacian, as proposed in $[26,36]$.

The perturbation method relies on a description in spatial or Eulerian coordinates. To that end, a compactum inside the domain is fixed and the domain deformation is considered relative to this compactum. The compactum has to be chosen in such a way that it is not intersected by the realizations of the domains boundary, cf. [18]. This particularly limits the magnitude of the boundary variation. The domain mapping method is based on a description in material or Lagrangian coordinates. Here, starting from the reference configuration $D_{\text {ref }}$, the trajectory of each particular point is tracked. In the domain mapping method, the notions of Eulerian and Lagrangian coordinates coincide on compacta, where the deformation is zero. Thus, in this sense, the domain mapping method provides the more general framework. The correspondence between the perturbation method and the domain mapping method can be expressed in terms of the local shape derivative $\delta u[\mathbf{V}(\omega)]$ and the material derivative $\dot{u}[\mathbf{V}(\omega)]$ of a given function $u$ which differ by a transport term, cf. [32]:

$$
\dot{u}[\mathbf{V}(\omega)]=\delta u[\mathbf{V}(\omega)]+\langle\nabla u, \mathbf{V}(\omega)\rangle .
$$

In this article, we focus on the domain mapping method. In [6], it is shown for a specific class of variation fields that the solution to (1) provides analytic regularity with respect to the random parameter. We will generalize the result from [6] to arbitrary domain perturbation fields which are described by their mean $\mathbb{E}[\mathbf{V}]: D_{\text {ref }} \rightarrow \mathbb{R}^{d}, \mathbb{E}[\mathbf{V}](\mathbf{x})=\left[\mathbb{E}\left[v_{1}\right](\mathbf{x}), \ldots, \mathbb{E}\left[v_{d}\right](\mathbf{x})\right]^{\top}$ and their (matrix-valued) covariance function

$$
\operatorname{Cov}[\mathbf{V}]: D_{\text {ref }} \times D_{\text {ref }} \rightarrow \mathbb{R}^{d \times d}, \quad \operatorname{Cov}[\mathbf{V}]\left(\mathbf{x}, \mathbf{x}^{\prime}\right)=\left[\begin{array}{cc}
\operatorname{Cov}_{1,1}\left(\mathbf{x}, \mathbf{x}^{\prime}\right) \cdots & \operatorname{Cov}_{1, d}\left(\mathbf{x}, \mathbf{x}^{\prime}\right) \\
\vdots & \vdots \\
\operatorname{Cov}_{d, 1}\left(\mathbf{x}, \mathbf{x}^{\prime}\right) \cdots & \operatorname{Cov}_{d, d}\left(\mathbf{x}, \mathbf{x}^{\prime}\right)
\end{array}\right]
$$


Note that the covariance function describes the covariance between any pair $\left(\mathbf{x}, \mathbf{x}^{\prime}\right)$ of points in $D_{\text {ref }}$ and induces thus a modeling in terms of Lagrangian coordinates. Taking the Karhunen-Loève expansion of $\mathbf{V}(\omega)$ as the starting point, we show decay rates for the derivatives of the solution to (1) with respect to the random parameter. Given that the Karhunen-Loève expansion decays fast enough, our results imply the dimension independent convergence of the quasi-Monte Carlo method based on the Halton sequence, cf. [13,16,34]. Moreover, our results are convenient for the convergence theory of the anisotropic sparse collocation, cf. [28], and best $N$-term approximations, cf. [8]. Although the presented results allow for a broad variety of methods for the stochastic approximation, we employ the quasiMonte Carlo method in our numerical examples for the sake of simplicity.

For the spatial approximation, we propose to use parametric finite elements. Then, we are able to approximate the mean and the variance of the solution to (1) by computing each sample on the particular realization $D\left(\omega_{i}\right)=\mathbf{V}\left(D_{\text {ref }}, \omega_{i}\right)$ of the random domain rather than on the reference domain $D_{\text {ref }}$. This yields a non-intrusive approach to solve the problem at hand. In fact, any available finite element solver can be employed to compute the particular samples. Following this approach rather than mapping the diffusion problem always to the reference domain, we can easily treat also stochastic interface problems, cf. [14].

The rest of this article is organized as follows. In Section 2, we introduce some basic definitions and notation. Section 3 is dedicated to the Karhunen-Loève expansion of vector fields. Although this is a straightforward adaption of the state of the art literature [29], we think that it is sensible to explicitly introduce the related spaces, norms and operators. In Section 4, we present the essential contribution of this article: the regularity of the solution to the model problem defined in Section 2 with respect to the Karhunen-Loève expansion of the perturbation field. Section 5 introduces parametric finite elements which are the basic ingredient for the numerical realization of our approach. In Section 6 , we extend our approach to stochastic interface problems. Finally, Section 7 provides numerical examples to validate and quantify the theoretical findings.

In the following, in order to avoid the repeated use of generic but unspecified constants, by $C \lesssim D$ we mean that $C$ can be bounded by a multiple of $D$, independently of parameters which $C$ and $D$ may depend on. Obviously, $C \gtrsim D$ is defined as $D \lesssim C$ and $C \approx D$ as $C \lesssim D$ and $C \gtrsim D$.

\section{Problem formulation}

Let $D_{\text {ref }} \subset \mathbb{R}^{d}$ for $d \in \mathbb{N}$ (of special interest are the cases $d=2,3$ ) denote a domain with Lipschitz continuous boundary $\partial D_{\text {ref }}$ and let $(\Omega, \mathcal{F}, \mathbb{P})$ be a complete probability space with $\sigma$-field $\mathcal{F} \subset 2^{\Omega}$ and probability measure $\mathbb{P}$. In order to guarantee that $L_{\mathbb{P}}^{2}(\Omega)$ exhibits an orthonormal basis, we further assume that $\Omega$ is a separable set. Let $\mathbf{V}: \overline{D_{\text {ref }}} \times \Omega \rightarrow \mathbb{R}^{d}$ be an invertible vector field of class $C^{2}$, i.e. $\mathbf{V}$ is twice continuously differentiable with respect to $\mathbf{x}$ for almost every $\omega \in \Omega$. Moreover, we impose the uniformity condition

$$
\|\mathbf{V}(\omega)\|_{C^{2}\left(\overline{D_{\text {ref }}} ; \mathbb{R}^{d}\right)},\left\|\mathbf{V}^{-1}(\omega)\right\|_{C^{2}\left(\overline{D_{\text {ref }}} ; \mathbb{R}^{d}\right)} \leq C
$$


for some $C \in(0, \infty)$ and almost every $\omega \in \Omega$. ${ }^{1}$ Thus, $\mathbf{V}$ defines a family of domains

$$
D(\omega):=\mathbf{V}\left(D_{\text {ref }}, \omega\right)
$$

For the subsequent analysis, we restrict ourselves to the case of the Poisson equation, i.e. $\alpha \equiv 1$,

$$
-\Delta u(\mathbf{x}, \omega)=f(\mathbf{x}) \text { in } D(\omega), \quad u(\mathbf{x}, \omega)=0 \text { on } \Gamma(\omega) .
$$

This considerably simplifies the analysis and the extension to non-constant diffusion coefficients is straightforward, cf. Remark 2. In order to guarantee solvability for almost every $\omega \in \Omega$, we consider the right hand side to be defined on the hold-all domain

$$
\mathcal{D}:=\bigcup_{\omega \in \Omega} D(\omega)
$$

From the uniformity condition, we infer for almost every $\omega \in \Omega$ and every $\mathbf{x} \in D$ that the singular-values of the vector field V's Jacobian $\mathbf{J}(\omega, \mathbf{x})$ satisfy

$$
0<\underline{\sigma} \leq \min \{\sigma(\mathbf{J}(\mathbf{x}, \omega))\} \leq \max \{\sigma(\mathbf{J}(\mathbf{x}, \omega))\} \leq \bar{\sigma}<\infty .
$$

In particular, we assume without loss of generality that $\underline{\sigma} \leq 1$ and $\bar{\sigma} \geq 1$.

\subsection{Reformulation on the reference domain}

In the sequel, we consider the spaces $H_{0}^{1}(D(\omega))$ and $H_{0}^{1}\left(D_{\text {ref }}\right)$ to be equipped with the norms $\|\cdot\|_{H^{1}(D(\omega))}:=\|\nabla \cdot\|_{L^{2}\left(D(\omega) ; \mathbb{R}^{d}\right)}$ and $\|\cdot\|_{H^{1}\left(D_{\mathrm{ref}}\right)}:=\|\nabla \cdot\|_{L^{2}\left(D_{\mathrm{ref}} ; \mathbb{R}^{d}\right)}$, respectively. Furthermore, we assume that the related dual spaces $H^{-1}(D(\omega))$ and $H^{-1}\left(D_{\text {ref }}\right)$ are defined with respect to these norms. The main tool we use in the convergence analysis for the model problem (2) is the one-to-one correspondence between the problem which is pulled back to the reference domain $D_{\text {ref }}$ and the problem on the actual realization $D(\omega)$. The equivalence between those two problems is described by the vector field $\mathbf{V}(\mathbf{x}, \omega)$. For an arbitrary function $v$ on $D(\omega)$, we denote the transported function by $\hat{v}(\mathbf{x}, \omega):=(v \circ \mathbf{V})(\mathbf{x}, \omega)$. According to the chain rule, we have for $v \in C^{1}(D(\omega))$

$$
(\nabla v)(\mathbf{V}(\mathbf{x}, \omega))=\mathbf{J}(\mathbf{x}, \omega)^{-\mathbf{T}} \nabla \hat{v}(\mathbf{x}, \omega) .
$$

For given $\omega \in \Omega$, the variational formulation for the model problem (2) reads as follows: Find $u(\omega) \in H_{0}^{1}(D(\omega))$ such that

$$
\int_{D(\omega)}\langle\nabla u, \nabla v\rangle \mathrm{d} \mathbf{x}=\int_{D(\omega)} f v \mathrm{~d} \mathbf{x} \quad \text { for all } v \in H_{0}^{1}(D(\omega)) .
$$

Thus, with

$$
\mathbf{A}(\mathbf{x}, \omega):=\left(\mathbf{J}(\mathbf{x}, \omega)^{\top} \mathbf{J}(\mathbf{x}, \omega)\right)^{-1} \operatorname{det} \mathbf{J}(\mathbf{x}, \omega)
$$

and

$$
f_{\text {ref }}(\mathbf{x}, \omega):=\hat{f}(\mathbf{x}, \omega) \operatorname{det} \mathbf{J}(\mathbf{x}, \omega),
$$

${ }^{1}$ Regard that for the analysis it is sufficient to assume that $\mathbf{V}$ is a $C^{1}$-diffeomorphism and satisfies the uniformity in $C^{1}\left(\overline{D_{\text {ref }}} ; \mathbb{R}^{d}\right)$. Nevertheless, in order to obtain $H^{2}$-regularity of the model problem, we make this stronger assumption. 
we obtain the following variational formulation with respect to the reference domain: Find $\hat{u}(\omega) \in H_{0}^{1}\left(D_{\text {ref }}\right)$ such that

$$
\int_{D_{\text {ref }}}\langle\mathbf{A}(\omega) \nabla \hat{u}(\omega), \nabla \hat{v}(\omega)\rangle \mathrm{d} \mathbf{x}=\int_{D_{\text {ref }}} f_{\text {ref }}(\omega) \hat{v}(\omega) \mathrm{d} \mathbf{x} \quad \text { for all } \hat{v}(\omega) \in H_{0}^{1}\left(D_{\text {ref }}\right) .
$$

Here and afterwards, $\langle\cdot, \cdot\rangle$ denotes the canonical inner product for $\mathbb{R}^{d}$.

Remark 1 Since $\mathbf{V}$ is assumed to be a $C^{2}$-diffeomorphism, we have for almost every $\omega \in \Omega$ that

$$
\mathbf{V}^{-1} \circ \mathbf{V}=\text { Id } \Rightarrow \mathbf{J}^{-1} \mathbf{J}=\mathbf{I} \Rightarrow \operatorname{det} \mathbf{J}^{-1} \operatorname{det} \mathbf{J}=1 \quad \text { for all } \mathbf{x} .
$$

Herein, $\mathbf{I} \in \mathbb{R}^{d \times d}$ denotes the identity matrix. Especially, we infer $\operatorname{det} \mathbf{J}^{-1}, \operatorname{det} \mathbf{J} \neq$ 0 . The continuity of $\mathbf{J}, \mathbf{J}^{-1}$ and of the determinant function imply now that either $\operatorname{det} \mathbf{J}^{-1}, \operatorname{det} \mathbf{J}>0$ or $\operatorname{det} \mathbf{J}^{-1}, \operatorname{det} \mathbf{J}<0$ for all $\mathbf{x}$. Therefore, without loss of generality, we will assume the positiveness of the determinants.

Notice that equation (9) contains for fixed $v \in H_{0}^{1}(D(\omega))$ the related transported test function $\hat{v}(\omega)$.

The connection between the spaces $H_{0}^{1}\left(D_{\text {ref }}\right)$ and $H_{0}^{1}(D(\omega))$ is given by the following

Lemma 1 The spaces $H_{0}^{1}\left(D_{\mathrm{ref}}\right)$ and $H_{0}^{1}(D(\omega))$ are isomorphic by the isomorphism

$$
\mathcal{E}: H_{0}^{1}\left(D_{\mathrm{ref}}\right) \rightarrow H_{0}^{1}(D(\omega)), \quad v \mapsto v \circ \mathbf{V}(\omega)^{-1}
$$

The inverse mapping is given by

$$
\mathcal{E}^{-1}: H_{0}^{1}(D(\omega)) \rightarrow H_{0}^{1}\left(D_{\text {ref }}\right), \quad v \mapsto v \circ \mathbf{V}(\omega)
$$

Proof The proof of this lemma is a consequence of the chain rule (5) and the ellipticity assumption (4).

This lemma implies that the space of test functions is not dependent on $\omega \in$ $\Omega$ at all: Obviously, we have $H_{0}^{1}(D(\omega))=\left\{\mathcal{E}(v): v \in H_{0}^{1}\left(D_{\text {ref }}\right)\right\}$. Thus, for an arbitrary function $\mathcal{E}(v) \in H_{0}^{1}(D(\omega))$ it holds $\widehat{\mathcal{E}(v)}=\mathcal{E}(v) \circ \mathbf{V}=v \circ \mathbf{V}^{-1} \circ \mathbf{V}=v \in$ $H_{0}^{1}\left(D_{\text {ref }}\right)$ independent of $\omega \in \Omega$. In particular, the solutions $u$ to (6) and $\hat{u}$ to (9) satisfy

$$
\hat{u}(\omega)=u \circ \mathbf{V}(\omega) \text { and } \quad u(\omega)=\hat{u} \circ \mathbf{V}(\omega)^{-1}
$$

\section{Karhunen-Loève expansion}

In order to make the random vector field $\mathbf{V}(\mathbf{x}, \omega)$ feasible for computations, we consider here its Karhunen-Loève expansion, cf. [25]. This section shall give a brief overview of the relevant facts concerning the Karhunen-Loève expansion of vector valued random fields. Especially, we introduce here the related function spaces which are used in the rest of this article. For further details on the KarhunenLoève expansion in general and also on computational aspects, we refer to $[10,11$, $17,29]$. 
Let $D \subset \mathbb{R}^{d}$ always denote a domain. Then, we define $L^{2}\left(D ; \mathbb{R}^{d}\right)$ to be the Hilbert space which consists of all equivalence classes of square integrable functions $\mathbf{v}: D \rightarrow \mathbb{R}^{d}$ equipped with the inner product

$$
(\mathbf{u}, \mathbf{v})_{L^{2}\left(D ; \mathbb{R}^{d}\right)}:=\int_{D}\langle\mathbf{u}, \mathbf{v}\rangle \mathrm{d} \mathbf{x} \quad \text { for all } \mathbf{u}, \mathbf{v} \in L^{2}\left(D ; \mathbb{R}^{d}\right)
$$

We assume that the vector field $\mathbf{V}$ satisfies

$$
\mathbf{V}(\mathbf{x}, \omega)=\left[v_{1}(\mathbf{x}, \omega), \ldots, v_{d}(\mathbf{x}, \omega)\right]^{\top} \in L_{\mathbb{P}}^{2}\left(\Omega ; L^{2}\left(D ; \mathbb{R}^{d}\right)\right)
$$

Here and in the sequel, given a Banach space $B$ and $1 \leq p \leq \infty$, the LebesgueBochner space $L_{\mathbb{P}}^{p}(\Omega ; B)$ consists of all equivalence classes of strongly measurable functions $v: \Omega \rightarrow B$ whose norm

$$
\|v\|_{L_{\mathbb{P}}^{p}(\Omega ; B)}:= \begin{cases}\left(\int_{\Omega}\|v(\cdot, \omega)\|_{B}^{p} \mathrm{~d} \mathbb{P}(\omega)\right)^{1 / p}, & p<\infty \\ \operatorname{ess~sup}_{\omega \in \Omega}\|v(\cdot, \omega)\|_{B}, & p=\infty\end{cases}
$$

is finite. If $B=H$ is a separable Hilbert space and $p=2$, then the LebesgueBochner space is isomorphic to the tensor product space $L_{\mathbb{P}}^{2}(\Omega) \otimes H$ equipped with the inner product

$$
(u, v)_{L_{\mathbb{P}}^{2}(\Omega ; H)}:=\int_{\Omega}(u(\cdot, \omega), v(\cdot, \omega))_{H} \mathrm{~d} \mathbb{P}(\omega),
$$

cf. $[2,24]$.

The mean of $\mathbf{V}$ is given by $\mathbb{E}[\mathbf{V}](\mathbf{x})=\left[\mathbb{E}\left[v_{1}\right](\mathbf{x}), \ldots, \mathbb{E}\left[v_{d}\right](\mathbf{x})\right]^{\top}$ with

$$
\mathbb{E}\left[v_{i}\right](\mathbf{x}):=\int_{\Omega} v_{i}(\mathbf{x}, \omega) \mathrm{d} \mathbb{P}(\omega), \quad i=1,2, \ldots, d .
$$

From the theory of Bochner integrals, see e.g. [24], it follows that $\mathbb{E}\left[v_{i}\right](\mathbf{x}) \in L^{2}(D)$ and thus $\mathbb{E}[\mathbf{V}](\mathbf{x}) \in L^{2}\left(D ; \mathbb{R}^{d}\right)$. Furthermore, the (matrix-valued) covariance function of $\mathbf{V}$ is given by $\operatorname{Cov}[\mathbf{V}](\mathbf{x}, \mathbf{y})=\left[\operatorname{Cov}_{i, j}(\mathbf{x}, \mathbf{y})\right]_{i, j=1}^{d}$ with

$$
\operatorname{Cov}_{i, j}(\mathbf{x}, \mathbf{y})=\mathbb{E}\left[\left(v_{i}(\mathbf{x}, \omega)-\mathbb{E}\left[v_{i}\right](\mathbf{x})\right)\left(v_{j}(\mathbf{y}, \omega)-\mathbb{E}\left[v_{j}\right](\mathbf{y})\right)\right] .
$$

We have $\operatorname{Cov}_{i, j}(\mathbf{x}, \mathbf{y}) \in L^{2}(D \times D)$ which also follows from the properties of the Bochner integral and the application of the Cauchy-Schwarz inequality. We therefore conclude $\operatorname{Cov}[\mathbf{V}](\mathbf{x}, \mathbf{y}) \in L^{2}\left(D \times D ; \mathbb{R}^{d \times d}\right)$ where we equip the space $\mathbb{R}^{d \times d}$ with the inner product

$$
\mathbf{A}: \mathbf{B}:=\sum_{i, j=1}^{d} a_{i, j} b_{i, j} \quad \text { for } \mathbf{A}, \mathbf{B} \in \mathbb{R}^{d \times d} \quad \text { with } \mathbf{A}=\left[a_{i, j}\right]_{i, j=1}^{d}, \quad \mathbf{B}=\left[b_{i, j}\right]_{i, j=1}^{d}
$$

This particularly induces the inner product on $L^{2}\left(D \times D ; \mathbb{R}^{d \times d}\right)$ given by

$$
(\mathbf{A}, \mathbf{B})_{L^{2}\left(D \times D ; \mathbb{R}^{d \times d}\right)}:=\int_{D} \int_{D} \mathbf{A}: \mathbf{B} \mathrm{d} \mathbf{x} \mathrm{d} \mathbf{y} \quad \text { for } \mathbf{A}, \mathbf{B} \in L^{2}\left(D \times D ; \mathbb{R}^{d \times d}\right) .
$$


Now, we shall introduce the operator

$$
\mathcal{S}: L_{\mathbb{P}}^{2}(\Omega) \rightarrow L^{2}\left(D ; \mathbb{R}^{d}\right), \quad(\mathcal{S} X)(\mathbf{x}):=\int_{\Omega}(\mathbf{V}(\mathbf{x}, \omega)-\mathbb{E}[\mathbf{V}](\mathbf{x})) X(\omega) \mathrm{d} \mathbb{P}(\omega)
$$

and its adjoint

$$
\mathcal{S}^{\star}: L^{2}\left(D ; \mathbb{R}^{d}\right) \rightarrow L_{\mathbb{P}}^{2}(\Omega), \quad\left(\mathcal{S}^{\star} \mathbf{u}\right)(\omega):=\int_{D}(\mathbf{V}(\mathbf{x}, \omega)-\mathbb{E}[\mathbf{V}](\mathbf{x}))^{\top} \mathbf{u}(\mathbf{x}) \mathrm{d} \mathbf{x} .
$$

Then, there holds the following

Lemma 2 The operators $\mathcal{S}$ and $\mathcal{S}^{\star}$ given by (11) and (12), respectively, are bounded with Hilbert-Schmidt norms $\|\mathcal{S}\|_{\mathrm{HS}}=\left\|\mathcal{S}^{\star}\right\|_{\mathrm{HS}}=\|\mathbf{V}-\mathbb{E}[\mathbf{V}]\|_{L_{\mathbb{P}}^{2}\left(\Omega ; L^{2}\left(D ; \mathbb{R}^{d}\right)\right)}$. Moreover, the covariance operator

$$
\mathcal{C}: L^{2}\left(D ; \mathbb{R}^{d}\right) \rightarrow L^{2}\left(D ; \mathbb{R}^{d}\right), \quad(\mathcal{C} \mathbf{v})(\mathbf{x}):=\int_{D} \operatorname{Cov}[\mathbf{V}](\mathbf{x}, \mathbf{y}) \mathbf{v}(\mathbf{y}) \mathrm{d} \mathbf{y}=\left(\mathcal{S S}^{\star} \mathbf{v}\right)(\mathbf{x})
$$

is a non-negative, symmetric, trace class operator with trace $\|\mathbf{V}-\mathbb{E}[\mathbf{V}]\|_{L_{\mathbb{P}}^{2}\left(\Omega ; L^{2}\left(D ; \mathbb{R}^{d}\right)\right)}^{2}$.

Proof The statement on the norms of $\mathcal{S}$ and $\mathcal{S}^{\star}$ follows by the application of Parseval's identity, see the last part of the proof. Moreover, we have for all $\mathbf{u} \in$ $L^{2}\left(D ; \mathbb{R}^{d}\right)$ that

$$
\begin{aligned}
\left(\mathcal{S S}^{\star} \mathbf{u}\right)(\mathbf{x}) & =\int_{\Omega}(\mathbf{V}(\mathbf{x}, \omega)-\mathbb{E}[\mathbf{V}](\mathbf{x})) \int_{D}(\mathbf{V}(\mathbf{y}, \omega)-\mathbb{E}[\mathbf{V}](\mathbf{y}))^{\top} \mathbf{u}(\mathbf{y}) \mathrm{d} \mathbf{y} \mathrm{d} \mathbb{P}(\omega) \\
& =\int_{D}\left(\int_{\Omega}(\mathbf{V}(\mathbf{x}, \omega)-\mathbb{E}[\mathbf{V}](\mathbf{x}))(\mathbf{V}(\mathbf{y}, \omega)-\mathbb{E}[\mathbf{V}](\mathbf{y}))^{\top} \mathrm{d} \mathbb{P}(\omega)\right) \mathbf{u}(\mathbf{y}) \mathrm{d} \mathbf{y} \\
& =\int_{D} \operatorname{Cov}[\mathbf{V}](\mathbf{x}, \mathbf{y}) \mathbf{u}(\mathbf{y}) \mathrm{d} \mathbf{y}=(\mathcal{C} \mathbf{u})(\mathbf{x}) .
\end{aligned}
$$

In particular, $\mathcal{C}$ is non-negative and symmetric according to

$$
(\mathcal{C} \mathbf{u}, \mathbf{u})_{L^{2}\left(D ; \mathbb{R}^{d}\right)}=\left(\mathcal{S}^{\star} \mathbf{u}, \mathcal{S}^{\star} \mathbf{u}\right)_{L_{\mathbb{P}}^{2}(\Omega)}=\left\|\mathcal{S}^{\star} \mathbf{u}\right\|_{L_{\mathbb{P}}^{2}(\Omega)}^{2} \geq 0 .
$$

Finally, to show that $\mathcal{C}$ is of trace class, let $\left\{\boldsymbol{\varphi}_{k}\right\}_{k}$ be an arbitrary orthonormal basis in $L^{2}\left(D ; \mathbb{R}^{d}\right)$. We thus have

$$
\begin{aligned}
\sum_{k}\left(\mathcal{C} \boldsymbol{\varphi}_{k}, \boldsymbol{\varphi}_{k}\right)_{L^{2}\left(D ; \mathbb{R}^{d}\right)} & =\sum_{k}\left\|\mathcal{S}^{\star} \boldsymbol{\varphi}_{k}\right\|_{L_{\mathbb{P}}^{2}(\Omega)}^{2}=\int_{\Omega} \sum_{k}\left(\mathcal{S}^{\star} \boldsymbol{\varphi}_{k}\right)^{2} \mathrm{~d} \mathbb{P}(\omega) \\
& =\int_{\Omega} \sum_{k}\left(\int_{D}(\mathbf{V}(\mathbf{x}, \omega)-\mathbb{E}[\mathbf{V}](\mathbf{x}))^{\top} \boldsymbol{\varphi}_{k} \mathrm{~d} \mathbf{x}\right)^{2} \mathrm{~d} \mathbb{P}(\omega) \\
& =\int_{\Omega} \int_{D}\langle\mathbf{V}(\mathbf{x}, \omega)-\mathbb{E}[\mathbf{V}](\mathbf{x}), \mathbf{V}(\mathbf{x}, \omega)-\mathbb{E}[\mathbf{V}](\mathbf{x})\rangle \mathrm{d} \mathbf{x} \mathrm{d} \mathbb{P}(\omega) \\
& =\|\mathbf{V}-\mathbb{E}[\mathbf{V}]\|_{L_{\mathbb{P}}^{2}\left(\Omega ; L^{2}\left(D ; \mathbb{R}^{d}\right)\right)}^{2},
\end{aligned}
$$

where we employed Parseval's identity in the second last step.

Trace class operators are especially compact, see e.g. [20,30], and exhibit hence a spectral decomposition. 
Theorem 1 Let $\mathcal{C}: L^{2}\left(D ; \mathbb{R}^{d}\right) \rightarrow L^{2}\left(D ; \mathbb{R}^{d}\right)$ be the covariance operator related to $\mathbf{V}(\mathbf{x}, \omega) \in L_{\mathbb{P}}^{2}\left(\Omega ; L^{2}\left(D ; \mathbb{R}^{d}\right)\right)$. Then, there exists an orthonormal set $\left\{\boldsymbol{\varphi}_{k}\right\}_{k}$ and a sequence $\lambda_{1} \geq \lambda_{2} \geq \ldots \geq 0$ such that $\mathcal{C} \boldsymbol{\varphi}_{k}=\lambda_{k} \boldsymbol{\varphi}_{k}$ for all $k=1,2, \ldots$ Furthermore, it holds

$$
\mathcal{C} \mathbf{u}=\sum_{k} \lambda_{k}\left(\mathbf{u}, \boldsymbol{\varphi}_{k}\right)_{L^{2}\left(D ; \mathbb{R}^{d}\right)} \boldsymbol{\varphi}_{k} \quad \text { for all } \mathbf{u} \in L^{2}\left(D ; \mathbb{R}^{d}\right)
$$

Proof For a proof of this theorem, we refer to [2].

We have now all prerequisites at hand to define the Karhunen-Loève expansion of the vector field $\mathbf{V}(\mathbf{x}, \omega) \in L_{\mathbb{P}}^{2}\left(\Omega ; L^{2}\left(D ; \mathbb{R}^{d}\right)\right)$.

Definition 1 Let $\mathbf{V}(\mathbf{x}, \omega)$ be a vector field in $L_{\mathbb{P}}^{2}\left(\Omega ; L^{2}\left(D ; \mathbb{R}^{d}\right)\right)$. The expansion

$$
\mathbf{V}(\mathbf{x}, \omega)=E[\mathbf{V}](\mathbf{x})+\sum_{k} \sigma_{k} \boldsymbol{\varphi}_{k}(\mathbf{x}) X_{k}(\omega)
$$

with $\sigma_{k}=\sqrt{\lambda_{k}}$ and $X_{k}=\mathcal{S}^{\star} \boldsymbol{\varphi}_{k} / \sigma_{k}$, where $\left\{\left(\lambda_{k}, \boldsymbol{\varphi}_{k}\right)\right\}_{k}$ is the sequence of eigenpairs of the underlying covariance operator $\mathcal{C}=\mathcal{S S}^{\star}$, is called Karhunen-Loève expansion of $\mathbf{V}(\mathbf{x}, \omega)$.

The space $L^{2}\left(D ; \mathbb{R}^{d}\right)$ served as pivot space for our considerations in the preceding derivation of the Karhunen-Loève expansion. In order to control the error of truncating the expansion after $M \in \mathbb{N}$ terms, i.e.

$$
\left\|\mathbf{V}(\mathbf{x}, \omega)-E[\mathbf{V}](\mathbf{x})-\sum_{k=1}^{M} \sigma_{k} \boldsymbol{\varphi}_{k}(\mathbf{x}) X_{k}(\omega)\right\|_{L^{2}\left(\Omega ; L^{2}\left(D ; \mathbb{R}^{d}\right)\right)}=\left(\sum_{k=M+1}^{\infty} \lambda_{k}\right)^{\frac{1}{2}}
$$

one has to study the decay of the singular values $\sigma_{k}$ in the representation (13). The particular rate of decay is known to depend on the spatial regularity of $\mathbf{V}(\mathbf{x}, \omega)$. To that end, we consider the Sobolev space $H^{p}\left(D ; \mathbb{R}^{d}\right)$ for $p>0$. The related inner product is given by

$$
(\mathbf{u}, \mathbf{w})_{H^{p}\left(D ; \mathbb{R}^{d}\right)}:=\sum_{|\boldsymbol{\alpha}| \leq p} \int_{D}\left\langle\partial^{\boldsymbol{\alpha}} \mathbf{u}, \partial^{\boldsymbol{\alpha}} \mathbf{w}\right\rangle \mathrm{d} \mathbf{x}
$$

for $p \in \mathbb{N}$ and

$$
(\mathbf{u}, \mathbf{w})_{H^{p}\left(D ; \mathbb{R}^{d}\right)}:=(\mathbf{u}, \mathbf{w})_{H\lfloor p\rfloor\left(D ; \mathbb{R}^{d}\right)}+\sum_{|\boldsymbol{\alpha}|=\lfloor p\rfloor} \int_{D} \int_{D} \frac{\left\|\partial^{\boldsymbol{\alpha}} \mathbf{u}(\mathbf{x})-\partial^{\boldsymbol{\alpha}} \mathbf{w}(\mathbf{y})\right\|_{2}^{2}}{\|\mathbf{x}-\mathbf{y}\|_{2}^{d+2 s}} \mathrm{~d} \mathbf{x} \mathrm{d} \mathbf{y}
$$

for $p=\lfloor p\rfloor+s$ with $s \in(0,1)$. Its dual space with respect to the $L^{2}$-duality pairing ist denoted as $\tilde{H}^{-p}\left(D ; \mathbb{R}^{d}\right)$.

For given $\mathbf{V}(\mathbf{x}, \omega) \in L_{\mathbb{P}}^{2}\left(\Omega ; H^{p}\left(D ; \mathbb{R}^{d}\right)\right)$, it obviously holds

$$
\operatorname{Cov}_{i, j}(\mathbf{x}, \mathbf{y}) \in H^{p}(D) \otimes H^{p}(D) \quad \text { for } i, j=1, \ldots, d,
$$

cf. [11]. Therefore, the following theorem is a straightforward modification of [11, Theorem 3.3] for the vector valued case.

Theorem 2 Let $\mathbf{V}(\mathbf{x}, \omega) \in L_{\mathbb{P}}^{2}\left(\Omega ; H^{p}\left(D ; \mathbb{R}^{d}\right)\right)$. Then, the eigenvalues of the covariance operator $\mathcal{C}: \tilde{H}^{-p}\left(D ; \mathbb{R}^{d}\right) \rightarrow H^{p}\left(D ; \mathbb{R}^{d}\right)$ decay like $\lambda_{k} \lesssim(k / d)^{-2 p / d}$ as $k \rightarrow \infty$. 
We may summarize the results of this section as follows. If the mean $\mathbb{E}[\mathbf{V}](\mathbf{x})$ and the covariance function $\operatorname{Cov}[\mathbf{V}](\mathbf{x}, \mathbf{y})$ as well as the distribution of $\mathbf{V}(\mathbf{x}, \omega)$ are known or appropriately estimated, cf. [29], we are able to reconstruct the vector field $\mathbf{V}(\mathbf{x}, \omega)$ from its Karhunen-Loève expansion. In the following, in order to make the Karhunen-Loève expansion feasible for computations, we make some common assumptions.

\section{Assumption 1}

(1) The random variables $\left\{X_{k}\right\}_{k}$ are centered and take values in $[-1,1]$, i.e. $X_{k}(\omega) \in$ $[-1,1]$ for all $k$ and almost every $\omega \in \Omega$.

(2) The random variables $\left\{X_{k}\right\}_{k}$ are independent and identically distributed.

(3) The sequence

$$
\left\{\gamma_{k}\right\}_{k}:=\left\{\left\|\sigma_{k} \boldsymbol{\varphi}_{k}\right\|_{W^{1, \infty}\left(D ; \mathbb{R}^{d}\right)}\right\}_{k}
$$

is at least in $\ell^{1}(\mathbb{N})$. We denote its $\ell^{1}$-norm by $c_{\gamma}:=\sum_{k=1}^{\infty} \gamma_{k}$.

Here and hereafter, we shall equip the space $W^{1, \infty}\left(D ; \mathbb{R}^{d}\right)$ with the equivalent norm $\|\mathbf{v}\|_{W^{1, \infty}\left(D ; \mathbb{R}^{d}\right)}=\max \left\{\|\mathbf{v}\|_{L^{\infty}\left(D ; \mathbb{R}^{d}\right)},\left\|\mathbf{v}^{\prime}\right\|_{L^{\infty}\left(D ; \mathbb{R}^{d \times d}\right)}\right\}$, where $\mathbf{v}^{\prime}$ denotes the Jacobian of $\mathbf{v}$ and $\left\|\mathbf{v}^{\prime}\right\|_{L^{\infty}\left(D ; \mathbb{R}^{d \times d}\right)}:=\operatorname{ess}_{\sup _{\mathbf{x} \in D}}\left\|\mathbf{v}^{\prime}(\mathbf{x})\right\|_{2}$. Herein, $\|\cdot\|_{2}$ is the usual 2 -norm of matrices, i.e. the largest singular value.

\section{Regularity of the solution}

In this section, we assume that the vector field $\mathbf{V}(\mathbf{x}, \mathbf{y})$ is given by a finite rank Karhunen-Loève expansion, i.e.

$$
\mathbf{V}(\mathbf{x}, \mathbf{y})=\mathbb{E}[\mathbf{V}](\mathbf{x})+\sum_{k=1}^{M} \sigma_{k} \boldsymbol{\varphi}_{k}(\mathbf{x}) y_{k}
$$

otherwise it has to be truncated appropriately. Nevertheless, we provide in this section estimates which are independent of $M \in \mathbb{N}$. Thus, we explicitly allow $M$ to become arbitrarily large.

For the rest of this article, we will refer to the randomness only via the coordinates $\mathbf{y} \in \square:=[-1,1]^{M}$, where $\mathbf{y}=\left[y_{1}, \ldots, y_{M}\right]$. Notice that due to the independence of the random variables, the related push-forward measure $\mathbb{P}_{\mathbf{X}}:=\mathbb{P} \circ \mathbf{X}^{-1}$ where $\mathbf{X}(\omega):=\left[X_{1}(\omega), \ldots X_{M}(\omega)\right]$ is of product structure. Furthermore, we always think of the spaces $L^{p}(\square)$ for $p \in[1, \infty]$ to be equipped with the measure $\mathbb{P}_{\mathbf{X}}$. Moreover, we set $\gamma=\left[\gamma_{k}\right]_{k=1}^{M}$, cf. (15).

Without loss of generality, we may assume that $\mathbb{E}[\mathbf{V}](\mathbf{x})=\mathbf{x}$ is the identity mapping. Otherwise, we replace $D_{\text {ref }}$ by

$$
\widetilde{D}_{\text {ref }}:=\mathbb{E}[\mathbf{V}]\left(D_{\text {ref }}\right) \quad \text { and } \quad \widetilde{\varphi}_{k}:=\sqrt{\operatorname{det}\left(\mathbb{E}[\mathbf{V}]^{-1}\right)^{\prime}} \varphi_{k} \circ \mathbb{E}[\mathbf{V}]^{-1} .
$$

Therefore, we obtain

$$
\mathbf{V}(\mathbf{x}, \mathbf{y})=\mathbf{x}+\sum_{k=1}^{M} \sigma_{k} \boldsymbol{\varphi}_{k}(\mathbf{x}) y_{k} \quad \text { and } \quad \mathbf{J}(\mathbf{x}, \mathbf{y})=\mathbf{I}+\sum_{k=1}^{M} \sigma_{k} \boldsymbol{\varphi}_{k}^{\prime}(\mathbf{x}) y_{k}
$$


In the subsequent regularity results, we shall refer to the following LebesgueBochner spaces. We define the space $L^{\infty}\left(\square ; L^{\infty}\left(D_{\text {ref }} ; \mathbb{R}^{d}\right)\right)$ as the set of all equivalence classes of strongly measurable functions $\mathbf{V}: \square \rightarrow L^{\infty}\left(D_{\text {ref }} ; \mathbb{R}^{d}\right)$ with finite norm

$$
\|\mathbf{V}\|_{d}:=\underset{\mathbf{y} \in \square}{\operatorname{ess} \sup }\|\mathbf{V}(\mathbf{y})\|_{L^{\infty}\left(D_{\mathrm{ref}} ; \mathbb{R}^{d}\right)}
$$

Furthermore, the space $L^{\infty}\left(\square ; L^{\infty}\left(D_{\text {ref }} ; \mathbb{R}^{d \times d}\right)\right)$ consists of all equivalence classes of strongly measurable functions $\mathbf{M}: \square \rightarrow L^{\infty}\left(D_{\text {ref }} ; \mathbb{R}^{d \times d}\right)$ with finite norm

$$
\|\mathbf{M}\|_{d \times d}:=\underset{\mathbf{y} \in \square}{\operatorname{ess} \sup }\|\mathbf{M}(\mathbf{y})\|_{L^{\infty}\left(D_{\text {ref }} ; \mathbb{R}^{d \times d}\right)} .
$$

We start by providing bounds on the derivatives of $\left(\mathbf{J}(\mathbf{x}, \mathbf{y})^{\top} \mathbf{J}(\mathbf{x}, \mathbf{y})\right)^{-1}$.

Lemma 3 Let $\mathbf{J}: D_{\text {ref }} \times \square \rightarrow \mathbb{R}^{d \times d}$ be defined as in (16). Then, it holds for the derivatives of

$$
\left(\mathbf{J}(\mathbf{x}, \mathbf{y})^{\top} \mathbf{J}(\mathbf{x}, \mathbf{y})\right)^{-1}
$$

under the conditions of Assumption 1.3 that

$$
\left\|\left|\partial_{\mathbf{y}}^{\boldsymbol{\alpha}}\left(\mathbf{J}^{\top} \mathbf{J}\right)^{-1}\right|\right\|_{d \times d} \leq|\boldsymbol{\alpha}| ! \frac{\gamma^{\boldsymbol{\alpha}}}{\underline{\sigma}^{2}}\left(\frac{2\left(1+c_{\gamma}\right)}{\underline{\sigma}^{2} \log 2}\right)^{|\boldsymbol{\alpha}|}
$$

Proof We define $\mathbf{B}(\mathbf{x}, \mathbf{y}):=\mathbf{J}(\mathbf{x}, \mathbf{y})^{\top} \mathbf{J}(\mathbf{x}, \mathbf{y})$ and $\tilde{\mathbf{A}}(\mathbf{x}, \mathbf{y}):=(\mathbf{B}(\mathbf{x}, \mathbf{y}))^{-1}$. Expanding the expression for $\mathbf{B}(\mathbf{x}, \mathbf{y})$ yields

$$
\mathbf{B}(\mathbf{x}, \mathbf{y})=\mathbf{I}+\sum_{k=1}^{M} \sigma_{k}\left(\boldsymbol{\varphi}_{k}^{\prime}(\mathbf{x})^{\top}+\varphi_{k}^{\prime}(\mathbf{x})\right) y_{k}+\sum_{k, k^{\prime}=1}^{M} \sigma_{k} \sigma_{k^{\prime}} \varphi_{k}^{\prime}(\mathbf{x})^{\top} \boldsymbol{\varphi}_{k^{\prime}}^{\prime}(\mathbf{x}) y_{k} y_{k^{\prime}} .
$$

Thus, the first order derivatives of $\mathbf{B}(\mathbf{x}, \mathbf{y})$ are given by

$$
\partial_{y_{i}} \mathbf{B}(\mathbf{x}, \mathbf{y})=\sigma_{i}\left(\boldsymbol{\varphi}_{i}^{\prime}(\mathbf{x})^{\top}+\boldsymbol{\varphi}_{i}^{\prime}(\mathbf{x})\right)+\sum_{k=1}^{M} \sigma_{i} \sigma_{k}\left(\boldsymbol{\varphi}_{i}^{\prime}(\mathbf{x})^{\top} \boldsymbol{\varphi}_{k}^{\prime}(\mathbf{x})+\boldsymbol{\varphi}_{k}^{\prime}(\mathbf{x})^{\top} \boldsymbol{\varphi}_{i}^{\prime}(\mathbf{x})\right) y_{k}
$$

and the second order derivatives according to

$$
\partial_{y_{j}} \partial_{y_{i}} \mathbf{B}(\mathbf{x}, \mathbf{y})=\sigma_{i} \sigma_{j}\left(\boldsymbol{\varphi}_{i}^{\prime}(\mathbf{x})^{\top} \boldsymbol{\varphi}_{j}^{\prime}(\mathbf{x})+\boldsymbol{\varphi}_{j}^{\prime}(\mathbf{x})^{\top} \boldsymbol{\varphi}_{i}^{\prime}(\mathbf{x})\right)
$$

Obviously, all higher order derivatives with respect to $\mathbf{y}$ vanish.

The ellipticity assumption (4) now yields the following bounds:

$$
\underline{\sigma}^{2} \leq\|\mathbf{B}\|_{d \times d} \leq \bar{\sigma}^{2} \quad \text { and } \quad \frac{1}{\bar{\sigma}^{2}} \leq\|\tilde{\mathbf{A}}\|_{d \times d} \leq \frac{1}{\underline{\sigma}^{2}},
$$

respectively. Furthermore, we derive from (17) that

$$
\left\|\partial_{y_{i}} \mathbf{B}\right\|_{d \times d} \leq 2 \gamma_{i}+2 \gamma_{i} \sum_{k=1}^{M} \gamma_{k} \leq 2\left(1+c_{\gamma}\right) \gamma_{i}
$$


and from (18) that $\left\|\partial_{y_{j}} \partial_{y_{i}} \mathbf{B}\right\| \|_{d \times d} \leq 2 \gamma_{i} \gamma_{j}$. Thus, we have

$$
\left\|\partial_{\mathbf{y}}^{\boldsymbol{\alpha}} \mathbf{B}\right\|_{d \times d} \leq \begin{cases}2\left(1+c_{\boldsymbol{\gamma}}\right) \boldsymbol{\gamma}^{\boldsymbol{\alpha}}, & \text { if }|\boldsymbol{\alpha}|=1,2 \\ 0, & \text { if }|\boldsymbol{\alpha}|>2\end{cases}
$$

Since $\tilde{\mathbf{A}}=v \circ \mathbf{B}$ is a composite function with $v(x)=x^{-1}$, we may employ Fà̀ di Bruno's formula, cf. [9], which is a generalization of the chain rule, to compute its derivatives. For $n=|\boldsymbol{\alpha}|$ Faà di Bruno's formula formally yields ${ }^{2}$

$$
\partial_{\mathbf{y}}^{\boldsymbol{\alpha}} \tilde{\mathbf{A}}(\mathbf{x}, \mathbf{y})=\sum_{r=1}^{n}(-1)^{r} r ! \tilde{\mathbf{A}}(\mathbf{x}, \mathbf{y})^{r+1} \sum_{P(\boldsymbol{\alpha}, r)} \boldsymbol{\alpha} ! \prod_{j=1}^{n} \frac{\left(\partial_{\mathbf{y}}^{\boldsymbol{\beta}_{j}} \mathbf{B}(\mathbf{x}, \mathbf{y})\right)^{k_{j}}}{k_{j} !\left(\boldsymbol{\beta}_{j} !\right)^{k_{j}}}
$$

Here, the set $P(\boldsymbol{\alpha}, r)$ contains restricted integer partitions of a multiindex $\boldsymbol{\alpha}$ into $r$ non-vanishing multiindices, i.e.

$$
\begin{array}{r}
P(\boldsymbol{\alpha}, r):=\left\{\left(\left(k_{1}, \boldsymbol{\beta}_{1}\right), \ldots,\left(k_{n}, \boldsymbol{\beta}_{n}\right)\right) \in\left(\mathbb{N}_{0} \times \mathbb{N}_{0}^{M}\right)^{n}: \sum_{i=1}^{n} k_{i} \boldsymbol{\beta}_{i}=\boldsymbol{\alpha}, \sum_{i=1}^{n} k_{i}=r,\right. \\
\text { and } \exists 1 \leq s \leq n: k_{i}=0 \text { and } \boldsymbol{\beta}_{i}=\mathbf{0} \text { for all } 1 \leq i \leq n-s, \\
\left.k_{i}>0 \text { for all } n-s+1 \leq i \leq n \text { and } \mathbf{0} \prec \boldsymbol{\beta}_{n-s+1} \prec \cdots \prec \boldsymbol{\beta}_{n}\right\} .
\end{array}
$$

Herein, for multiindices $\boldsymbol{\beta}, \boldsymbol{\beta}^{\prime} \in \mathbb{N}_{0}^{M}$, the relation $\boldsymbol{\beta} \prec \boldsymbol{\beta}^{\prime}$ means either $|\boldsymbol{\beta}|<\left|\boldsymbol{\beta}^{\prime}\right|$ or, if $|\boldsymbol{\beta}|=\left|\boldsymbol{\beta}^{\prime}\right|$, it denotes the lexicographical order which means that it holds that $\beta_{1}=\beta_{1}^{\prime}, \ldots, \beta_{k}=\beta_{k}^{\prime}$ and $\beta_{k+1}<\beta_{k+1}^{\prime}$ for some $0 \leq k<m$.

Taking the norm in (20), we derive the estimate

$$
\begin{aligned}
\left\|\partial_{\mathbf{y}}^{\boldsymbol{\alpha}} \tilde{\mathbf{A}}\right\| \|_{d \times d} & \leq \sum_{r=1}^{n} r !\|\tilde{\mathbf{A}}\|_{d \times d}^{r+1} \sum_{P(\boldsymbol{\alpha}, r)} \boldsymbol{\alpha} ! \prod_{j=1}^{n} \frac{\left\|\partial_{\mathbf{y}}^{\boldsymbol{\beta}_{j}} \mathbf{B}\right\|_{d \times d}^{k_{j}}}{k_{j} !\left(\boldsymbol{\beta}_{j} !\right)^{k_{j}}} \\
& \leq \sum_{r=1}^{n} r !\left(\frac{1}{\underline{\sigma}^{2}}\right)^{r+1} \sum_{P(\boldsymbol{\alpha}, r)} \boldsymbol{\alpha} ! \prod_{j=1}^{n} \frac{\left(2\left(1+c_{\boldsymbol{\gamma}}\right) \boldsymbol{\gamma}^{\boldsymbol{\beta}_{j}}\right)^{k_{j}}}{k_{j} !\left(\boldsymbol{\beta}_{j} !\right)^{k_{j}}} \\
& =\boldsymbol{\gamma}^{\boldsymbol{\alpha}} \sum_{r=1}^{n} r !\left(\frac{1}{\underline{\sigma}^{2}}\right)^{r+1}\left(2\left(1+c_{\boldsymbol{\gamma}}\right)\right)^{r} \sum_{P(\boldsymbol{\alpha}, r)} \boldsymbol{\alpha} ! \prod_{j=1}^{n} \frac{1}{k_{j} !\left(\boldsymbol{\beta}_{j} !\right)^{k_{j}}}
\end{aligned}
$$

From [9] we know that

$$
\sum_{P(\boldsymbol{\alpha}, r)} \boldsymbol{\alpha} ! \prod_{j=1}^{n} \frac{1}{k_{j} !\left(\boldsymbol{\beta}_{j} !\right)^{k_{j}}}=S_{n, r}
$$

where $S_{n, r}$ are the Stirling numbers of the second kind, cf. [1]. Thus, we obtain

$$
\left\|\partial_{\mathbf{y}}^{\boldsymbol{\alpha}} \tilde{\mathbf{A}}\right\|_{d \times d} \leq \frac{\gamma^{\boldsymbol{\alpha}}}{{\underline{\sigma^{2}}}^{2}} \sum_{r=1}^{n} r !\left(\frac{2\left(1+c_{\gamma}\right)}{\underline{\sigma}^{2}}\right)^{r} S_{n, r} \leq \frac{\gamma^{\boldsymbol{\alpha}}}{{\underline{\sigma^{2}}}^{2}}\left(\frac{2\left(1+c_{\boldsymbol{\gamma}}\right)}{\underline{\sigma}^{2}}\right)^{|\boldsymbol{\alpha}|} \sum_{r=1}^{n} r ! S_{n, r} .
$$

2 With "formally" we mean that we ignore here the fact that the product of matrices is in general not Abelian. Nevertheless, a differentiation yields exactly the appearing products in a permuted order. The formal representation is justified since we only consider the norm of the representation in the sequel. 
The term $\tilde{b}(n):=\sum_{r=0}^{n} r ! S_{n, r}$ coincides with the $n$-th ordered Bell number. The ordered Bell numbers satisfy the recurrence relation

$$
\tilde{b}(n)=\sum_{r=0}^{n-1}\left(\begin{array}{l}
n \\
k
\end{array}\right) \tilde{b}(r) \quad \text { with } \tilde{b}(0)=1,
$$

see [12], and may be estimated as follows ${ }^{3}$, cf. [3],

$$
\tilde{b}(n) \leq \frac{n !}{(\log 2)^{n}} .
$$

This finally proves the assertion.

The next lemma bounds the derivatives of $\operatorname{det} \mathbf{J}(\mathbf{x}, \mathbf{y})$.

Lemma 4 Let $\mathbf{J}: \square \rightarrow L^{\infty}\left(D_{\text {ref }} ; \mathbb{R}^{d \times d}\right)$ be defined as in (16). Then, it holds for the derivatives of $\operatorname{det} \mathbf{J}(\mathbf{x}, \mathbf{y})$ that

$$
\left\|\partial_{\mathbf{y}}^{\boldsymbol{\alpha}} \operatorname{det} \mathbf{J}(\mathbf{x}, \mathbf{y})\right\|_{L^{\infty}\left(\square ; L^{\infty}\left(D_{\text {ref }}\right)\right)} \leq d !(1+\bar{\sigma})^{d}|\boldsymbol{\alpha}| ! \gamma^{\boldsymbol{\alpha}} .
$$

Proof The proof is by induction on the minors of $\mathbf{J}(\mathbf{x}, \mathbf{y})=\left[j(\mathbf{x}, \mathbf{y})_{k, \ell}\right]_{k, \ell=1}^{d} \in \mathbb{R}^{d \times d}$. For the $(1 \times 1)$-minors, we obviously obtain

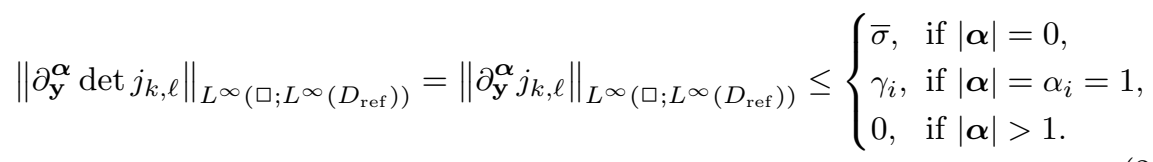

For $m \leq d$, we set $\mathbf{J}^{\mathbf{k}, \ell}:=\left[j_{k, \ell}\right]_{k \in \mathbf{k}, \ell \in \ell} \in \mathbb{R}^{m \times m}$, where $\mathbf{k}=\left\{k_{1}, \ldots, k_{m}\right\}$ and $\ell=\left[\ell_{1}, \ldots, \ell_{m}\right]$ with $1 \leq k_{1}<\ldots<k_{m} \leq d$ and $1 \leq \ell_{1}<\ldots<\ell_{m} \leq d$. Now, let the assertion (23) hold for some $m-1<d$. Then, Laplace's rule for determinants yields

$$
\left\|\partial_{\mathbf{y}}^{\boldsymbol{\alpha}} \operatorname{det} \mathbf{J}^{\mathbf{k}, \ell}\right\|_{L^{\infty}\left(\square ; L^{\infty}\left(D_{\mathrm{ref}}\right)\right)}=\left\|\partial_{\mathbf{y}}^{\boldsymbol{\alpha}} \sum_{\ell^{\prime}=1}^{m}(-1)^{k^{\prime}+\ell^{\prime}} j_{k_{k^{\prime}}, \ell_{\ell^{\prime}}} \operatorname{det} \mathbf{J}^{\mathbf{k}^{\prime}, \ell^{\prime}}\right\|_{L^{\infty}\left(\square ; L^{\infty}\left(D_{\mathrm{ref}}\right)\right)},
$$

where $\mathbf{k}^{\prime}:=\mathbf{k} \backslash\left\{k_{k^{\prime}}\right\}$ and $\ell^{\prime}:=\boldsymbol{\ell} \backslash\left\{\ell_{\ell^{\prime}}\right\}$. The triangle inequality and Leibniz rule for differentiation give us

$$
\begin{aligned}
& \left\|\partial_{\mathbf{y}}^{\boldsymbol{\alpha}} \sum_{k^{\prime}=1}^{m}(-1)^{k^{\prime}+\ell^{\prime}} j_{k_{k^{\prime}}, \ell_{\ell^{\prime}}} \operatorname{det} \mathbf{J}^{\mathbf{k}^{\prime}, \ell^{\prime}}\right\|_{L^{\infty}\left(\square ; L^{\infty}\left(D_{\mathrm{ref}}\right)\right)} \\
& \quad \leq \sum_{k^{\prime}=1}^{m}\left\|\partial_{\mathbf{y}}^{\boldsymbol{\alpha}}\left(j_{k_{k^{\prime}}, \ell_{\ell^{\prime}}} \operatorname{det} \mathbf{J}^{\mathbf{k}^{\prime}, \ell^{\prime}}\right)\right\|_{L^{\infty}\left(\square ; L^{\infty}\left(D_{\mathrm{ref}}\right)\right)} \\
& \quad=\sum_{k^{\prime}=1}^{m}\left\|\sum_{\boldsymbol{\alpha}^{\prime} \leq \boldsymbol{\alpha}}\left(\begin{array}{c}
\boldsymbol{\alpha} \\
\boldsymbol{\alpha}^{\prime}
\end{array}\right) \partial_{\mathbf{y}}^{\boldsymbol{\alpha}^{\prime}} j_{k_{k^{\prime}}, \ell_{\ell^{\prime}}} \partial_{\mathbf{y}}^{\boldsymbol{\alpha}-\boldsymbol{\alpha}^{\prime}} \operatorname{det} \mathbf{J}^{\mathbf{k}^{\prime}, \ell^{\prime}}\right\|_{L^{\infty}\left(\square ; L^{\infty}\left(D_{\mathrm{ref}}\right)\right)} \\
& \quad=\sum_{k^{\prime}=1}^{m}\left\|\sum_{r=1}^{M} \alpha_{r} \partial_{\mathbf{y}}^{\mathbf{e}_{r}} j_{k_{k^{\prime}}, \ell_{\ell^{\prime}}} \partial_{\mathbf{y}}^{\boldsymbol{\alpha}-\mathbf{e}_{r}} \operatorname{det} \mathbf{J}^{\mathbf{k}^{\prime}, \ell^{\prime}}+j_{k_{k^{\prime}}, \ell_{\ell^{\prime}}} \partial_{\mathbf{y}}^{\boldsymbol{\alpha}} \operatorname{det} \mathbf{J}^{\mathbf{k}^{\prime}, \ell^{\prime}}\right\|_{L^{\infty}\left(\square ; L^{\infty}\left(D_{\mathrm{ref}}\right)\right)},
\end{aligned}
$$

3 A more rigorous bound on the ordered Bell numbers is provided by [35]. There, it is shown that

$$
\tilde{b}(n)=\frac{n !}{2(\log 2)^{n+1}}+\mathcal{O}\left((0.16)^{n} n !\right) .
$$

Nevertheless, for our purposes, the bound from [3] is sufficient. 
since $j_{k_{k^{\prime}}, \ell_{\ell^{\prime}}}$ is an affine function with respect to $\mathbf{y}$ and all higher order derivatives, i.e. $\left|\boldsymbol{\alpha}^{\prime}\right|>1$, vanish, see (24). A reapplication of the triangle inequality together with the induction hypothesis and the sub-multiplicativity of the $L^{\infty}$-norm hence provides

$$
\begin{aligned}
& \sum_{k^{\prime}=1}^{m}\left\|\sum_{r=1}^{M} \alpha_{r} \partial_{\mathbf{y}}^{\mathbf{e}_{r}} j_{k_{k^{\prime}}, \ell_{\ell^{\prime}}} \partial_{\mathbf{y}}^{\boldsymbol{\alpha}-\mathbf{e}_{r}} \operatorname{det} \mathbf{J}^{\mathbf{k}^{\prime}, \ell^{\prime}}+j_{k_{k^{\prime}}, \ell_{\ell^{\prime}}} \partial_{\mathbf{y}}^{\boldsymbol{\alpha}} \operatorname{det} \mathbf{J}^{\mathbf{k}^{\prime}, \ell^{\prime}}\right\|_{L^{\infty}\left(\square ; L^{\infty}\left(D_{\mathrm{ref}}\right)\right)} \\
& \leq \sum_{k^{\prime}=1}^{m}\left(\sum_{r=1}^{M} \alpha_{r}\left\|\partial_{\mathbf{y}^{r}}^{\mathbf{e}_{r}} j_{k_{k^{\prime}}, \ell_{\ell^{\prime}}}\right\|_{L^{\infty}\left(\square ; L^{\infty}\left(D_{\mathrm{ref}}\right)\right)}\left\|\partial_{\mathbf{y}}^{\boldsymbol{\alpha}-\mathbf{e}_{i}} \operatorname{det} \mathbf{J}^{\mathbf{k}^{\prime}, \ell^{\prime}}\right\|_{L^{\infty}\left(\square ; L^{\infty}\left(D_{\mathrm{ref}}\right)\right)}\right) \\
& \left.\quad+\left\|j_{k_{k^{\prime}}, \ell_{\ell^{\prime}}}\right\|_{L^{\infty}\left(\square ; L^{\infty}\left(D_{\mathrm{ref}}\right)\right)}\left\|\partial_{\mathbf{y}}^{\boldsymbol{\alpha}} \operatorname{det} \mathbf{J}^{\mathbf{k}^{\prime}, \ell^{\prime}}\right\|_{L^{\infty}\left(\square ; L^{\infty}\left(D_{\mathrm{ref}}\right)\right)}\right) \\
& \quad \sum_{k^{\prime}=1}^{m}\left(\sum_{r=1}^{M} \alpha_{r} \gamma_{r}(m-1) !(1+\bar{\sigma})^{m-1}\left|\boldsymbol{\alpha}-\mathbf{e}_{r}\right| ! \boldsymbol{\gamma}^{\boldsymbol{\alpha}-\mathbf{e}_{r}}\right. \\
& \left.\quad+\bar{\sigma}(m-1) !(1+\bar{\sigma})^{m-1}|\boldsymbol{\alpha}| ! \boldsymbol{\gamma}^{\boldsymbol{\alpha}}\right) \\
& \leq \sum_{k^{\prime}=1}^{m}(m-1) !(1+\bar{\sigma})^{m-1}|\boldsymbol{\alpha}| ! \boldsymbol{\gamma}^{\boldsymbol{\alpha}}+\bar{\sigma}(m-1) !(1+\bar{\sigma})^{m-1}|\boldsymbol{\alpha}| ! \boldsymbol{\gamma}^{\boldsymbol{\alpha}} \\
& =m !(1+\bar{\sigma})^{m}|\boldsymbol{\alpha}| ! \boldsymbol{\gamma}^{\boldsymbol{\alpha}},
\end{aligned}
$$

where we exploited that

$$
\sum_{r=1}^{M} \alpha_{r}\left|\boldsymbol{\alpha}-\mathbf{e}_{r}\right| !=(|\boldsymbol{\alpha}|-1) ! \sum_{r=1}^{M} \alpha_{r}=(|\boldsymbol{\alpha}|-1) !|\boldsymbol{\alpha}|=|\boldsymbol{\alpha}| !
$$

The application of the Leibniz rule yields now a regularity estimate for the diffusion matrix $\mathbf{A}(\mathbf{x}, \mathbf{y})$.

Theorem 3 The derivatives of the diffusion matrix $\mathbf{A}(\mathbf{x}, \mathbf{y})$ defined in (7) satisfy under the conditions of Assumption1.3 that

$$
\left\|\partial_{\mathbf{y}}^{\boldsymbol{\alpha}} \mathbf{A}\right\|_{d \times d} \leq(|\boldsymbol{\alpha}|+1) ! \frac{C_{\mathrm{det}}}{\underline{\sigma}^{2}}\left(\frac{2\left(1+c_{\gamma}\right)}{\underline{\sigma}^{2} \log 2}\right)^{|\boldsymbol{\alpha}|} \boldsymbol{\gamma}^{\boldsymbol{\alpha}}
$$

Proof The Leibniz rule for $\partial_{\mathbf{y}}^{\boldsymbol{\alpha}} \mathbf{A}(\mathbf{x}, \mathbf{y})$ reads as

$$
\partial_{\mathbf{y}}^{\boldsymbol{\alpha}} \mathbf{A}(\mathbf{x}, \mathbf{y})=\sum_{\boldsymbol{\alpha}^{\prime} \leq \boldsymbol{\alpha}}\left(\begin{array}{c}
\boldsymbol{\alpha} \\
\boldsymbol{\alpha}^{\prime}
\end{array}\right) \partial_{\mathbf{y}}^{\boldsymbol{\alpha}^{\prime}}\left(\mathbf{J}(\mathbf{x}, \mathbf{y})^{\top} \mathbf{J}(\mathbf{x}, \mathbf{y})\right)^{-1} \partial_{\mathbf{y}}^{\boldsymbol{\alpha}-\boldsymbol{\alpha}^{\prime}} \operatorname{det} \mathbf{J}(\mathbf{x}, \mathbf{y}) .
$$

Inserting the results of Lemma 3 and Lemma 4 yields with $C_{\text {det }}:=d !(1+\bar{\sigma})^{d}$ that

$$
\begin{aligned}
& \left\|\partial_{\mathbf{y}}^{\boldsymbol{\alpha}} \mathbf{A}\right\|_{d \times d} \\
& \quad \leq \sum_{\boldsymbol{\alpha}^{\prime} \leq \boldsymbol{\alpha}}\left(\begin{array}{c}
\boldsymbol{\alpha} \\
\boldsymbol{\alpha}^{\prime}
\end{array}\right)\left|\boldsymbol{\alpha}^{\prime}\right| ! \frac{\boldsymbol{\gamma}^{\boldsymbol{\alpha}^{\prime}}}{{\underline{\sigma^{2}}}^{2}}\left(\frac{2\left(1+c_{\boldsymbol{\gamma}}\right)}{\underline{\sigma}^{2} \log 2}\right)^{\left|\boldsymbol{\alpha}^{\prime}\right|} C_{\mathrm{det}}\left|\boldsymbol{\alpha}-\boldsymbol{\alpha}^{\prime}\right| ! \boldsymbol{\gamma}^{\boldsymbol{\alpha}-\boldsymbol{\alpha}^{\prime}} \\
& \leq \frac{C_{\mathrm{det}}}{\underline{\sigma}^{2}}\left(\frac{2\left(1+c_{\boldsymbol{\gamma}}\right)}{\underline{\sigma}^{2} \log 2}\right)^{|\boldsymbol{\alpha}|} \boldsymbol{\gamma}^{\boldsymbol{\alpha}} \sum_{\boldsymbol{\alpha}^{\prime} \leq \boldsymbol{\alpha}}\left(\begin{array}{c}
\boldsymbol{\alpha} \\
\boldsymbol{\alpha}^{\prime}
\end{array}\right)\left|\boldsymbol{\alpha}^{\prime}\right| !\left|\boldsymbol{\alpha}-\boldsymbol{\alpha}^{\prime}\right| !
\end{aligned}
$$


Now, we employ the combinatorial identity

$$
\sum_{\substack{\boldsymbol{\alpha}^{\prime} \leq \boldsymbol{\alpha} \\
\left|\boldsymbol{\alpha}^{\prime}\right|=j}}\left(\begin{array}{c}
\boldsymbol{\alpha} \\
\boldsymbol{\alpha}^{\prime}
\end{array}\right)=\left(\begin{array}{c}
|\boldsymbol{\alpha}| \\
j
\end{array}\right)
$$

and obtain

$$
\begin{array}{r}
\sum_{\boldsymbol{\alpha}^{\prime} \leq \boldsymbol{\alpha}}\left(\begin{array}{c}
\boldsymbol{\alpha} \\
\boldsymbol{\alpha}^{\prime}
\end{array}\right)\left|\boldsymbol{\alpha}^{\prime}\right| !\left|\boldsymbol{\alpha}-\boldsymbol{\alpha}^{\prime}\right| !=\sum_{j=0}^{|\boldsymbol{\alpha}|} j !(|\boldsymbol{\alpha}|-j) ! \sum_{\substack{\boldsymbol{\alpha}^{\prime} \leq \boldsymbol{\alpha} \\
\left|\boldsymbol{\alpha}^{\prime}\right|=j}}\left(\begin{array}{c}
\boldsymbol{\alpha} \\
\boldsymbol{\alpha}^{\prime}
\end{array}\right) \\
=\sum_{j=0}^{|\boldsymbol{\alpha}|} j !(|\boldsymbol{\alpha}|-j) !\left(\begin{array}{c}
|\boldsymbol{\alpha}| \\
j
\end{array}\right)=|\boldsymbol{\alpha}| ! \sum_{j=0}^{|\boldsymbol{\alpha}|} 1=(|\boldsymbol{\alpha}|+1) !
\end{array}
$$

In order to prove regularity results for the right hand side $f_{\text {ref }}$ in (9), we have to assume that $f$ is a smooth function.

Lemma 5 Let $f \in C^{\infty}(\mathcal{D})$ be analytic, i.e. $\left\|\partial_{\mathbf{x}}^{\boldsymbol{\alpha}} f\right\|_{L^{\infty}\left(\mathcal{D} ; \mathbb{R}^{d}\right)} \leq \boldsymbol{\alpha} ! \rho^{-|\boldsymbol{\alpha}|} c_{f}$ for all $\boldsymbol{\alpha} \in$ $\mathbb{N}_{0}^{d}$ and some $\rho \in(0,1]$. Then, the derivatives of $\hat{f}=f \circ \mathbf{V}$ are bounded by

$$
\left\|\partial_{\mathbf{y}}^{\boldsymbol{\alpha}} \hat{f}\right\|_{L^{\infty}\left(\square ; L^{\infty}\left(D_{\mathrm{ref}}\right)\right)} \leq|\boldsymbol{\alpha}| ! c_{f}\left(\frac{d}{\rho \log 2}\right)^{|\boldsymbol{\alpha}|} \boldsymbol{\gamma}^{\boldsymbol{\alpha}} .
$$

Proof In view of (16), differentiation of $\mathbf{V}(\mathbf{x}, \mathbf{y})$ yields $\partial_{y_{i}} \mathbf{V}(\mathbf{x}, \mathbf{y})=\sigma_{i} \boldsymbol{\varphi}_{i}(\mathbf{x})$. Thus, all higher order derivatives with respect to an arbitrary direction $y_{j}$ vanish. The norm of the first order derivatives is bounded by $\left\|\partial_{y_{i}} \mathbf{V}\right\| \|_{d} \leq \gamma_{i}$.

The rest of the proof is also based on the application of Faà di Bruno's formula. Nevertheless, we have this time to consider the multivariate case. To that end, we define the set $P\left(\boldsymbol{\alpha}, \boldsymbol{\alpha}^{\prime}\right)$ given by

$$
\begin{gathered}
P\left(\boldsymbol{\alpha}, \boldsymbol{\alpha}^{\prime}\right):=\left\{\left(\left(\mathbf{k}_{1}, \boldsymbol{\beta}_{1}\right), \ldots,\left(\mathbf{k}_{n}, \boldsymbol{\beta}_{n}\right)\right) \in\left(\mathbb{N}_{0}^{d} \times \mathbb{N}_{0}^{M}\right)^{n}: \sum_{i=1}^{n}\left|\mathbf{k}_{i}\right| \boldsymbol{\beta}_{i}=\boldsymbol{\alpha}, \sum_{i=1}^{n} \mathbf{k}_{i}=\boldsymbol{\alpha}^{\prime},\right. \\
\text { and } \exists 1 \leq s \leq n:\left|\mathbf{k}_{j}\right|=\left|\boldsymbol{\beta}_{a}\right|=0 \text { for all } 1 \leq i \leq n-s, \\
\left.\quad\left|\mathbf{k}_{i}\right| \neq 0 \text { for all } n-s+1 \leq i \leq n \text { and } \mathbf{0} \prec \boldsymbol{\beta}_{n-s+1} \prec \cdots \prec \boldsymbol{\beta}_{n}\right\}
\end{gathered}
$$

with $n=|\boldsymbol{\alpha}|$. The application of the multivariate Faà di Bruno formula yields now

$$
\begin{aligned}
& \left\|\partial_{\mathbf{y}}^{\boldsymbol{\alpha}} \hat{f}\right\|_{L^{\infty}\left(\square ; L^{\infty}\left(D_{\mathrm{ref}}\right)\right)} \\
& \quad \leq \sum_{1 \leq\left|\boldsymbol{\alpha}^{\prime}\right| \leq n}\left\|\partial_{\mathbf{x}}^{\boldsymbol{\alpha}^{\prime}} f\right\|_{L^{\infty}\left(\square ; L^{\infty}(\mathcal{D})\right)} \sum_{P\left(\boldsymbol{\alpha}, \boldsymbol{\alpha}^{\prime}\right)} \boldsymbol{\alpha} ! \prod_{j=1}^{n} \frac{\left\|\left(\partial_{\mathbf{y}}^{\boldsymbol{\beta}_{j}} \mathbf{V}\right)^{\mathbf{k}_{j}}\right\|_{L^{\infty}\left(\square ; L^{\infty}\left(D_{\mathrm{ref}}\right)\right)}}{\mathbf{k}_{j} !\left(\boldsymbol{\beta}_{j} !\right)^{\left|\mathbf{k}_{j}\right|}} \\
& \quad \leq \sum_{1 \leq\left|\boldsymbol{\alpha}^{\prime}\right| \leq n} \boldsymbol{\alpha}^{\prime} ! \rho^{-\left|\boldsymbol{\alpha}^{\prime}\right|} c_{f} \sum_{P\left(\boldsymbol{\alpha}, \boldsymbol{\alpha}^{\prime}\right)} \boldsymbol{\alpha} ! \prod_{j=1}^{n} \frac{\left(\boldsymbol{\gamma}^{\left.\boldsymbol{\beta}_{j}\right)^{\mathbf{k}_{j}}}\right.}{\mathbf{k}_{j} !\left(\boldsymbol{\beta}_{j} !\right)^{\left|\mathbf{k}_{j}\right|}} \\
& \quad=c_{f} \boldsymbol{\gamma}^{\boldsymbol{\alpha}} \sum_{1 \leq\left|\boldsymbol{\alpha}^{\prime}\right| \leq n} \boldsymbol{\alpha}^{\prime} ! \rho^{-\left|\boldsymbol{\alpha}^{\prime}\right|} \sum_{P\left(\boldsymbol{\alpha}, \boldsymbol{\alpha}^{\prime}\right)} \boldsymbol{\alpha} ! \prod_{j=1}^{n} \frac{1}{\mathbf{k}_{j} !\left(\boldsymbol{\beta}_{j} !\right)^{\left|\mathbf{k}_{j}\right|}} .
\end{aligned}
$$


From [9], we know that

$$
\sum_{\left|\boldsymbol{\alpha}^{\prime}\right|=r} \sum_{P\left(\boldsymbol{\alpha}, \boldsymbol{\alpha}^{\prime}\right)} \boldsymbol{\alpha} ! \prod_{j=1}^{n} \frac{1}{\mathbf{k}_{j} !\left(\boldsymbol{\beta}_{j} !\right)^{\left|\mathbf{k}_{j}\right|}}=d^{r} S_{n, r}
$$

where again $S_{n, r}$ is the Stirling number of the second kind. Thus, we obtain

$$
\left\|\partial_{\mathbf{y}}^{\boldsymbol{\alpha}} \hat{f}\right\|_{L^{\infty}\left(\square ; L^{\infty}\left(D_{\mathrm{ref}}\right)\right)} \leq c_{f} \boldsymbol{\gamma}^{\boldsymbol{\alpha}} \sum_{r=1}^{n}\left(\frac{d}{\rho}\right)^{r} r ! S_{n, r} \leq c_{f} \boldsymbol{\gamma}^{\boldsymbol{\alpha}}\left(\frac{d}{\rho}\right)^{|\boldsymbol{\alpha}|} \sum_{r=0}^{n} r ! S_{n, r} .
$$

Analogously to the proof of Lemma 3, we finally arrive at the assertion.

Now, in complete analogy to Theorem 3, we have the following regularity result for the right hand side $f_{\text {ref }}$.

Theorem 4 The derivatives of the right hand side $f_{\mathrm{ref}}(\mathbf{x}, \mathbf{y})$ defined in (8) satisfy

$$
\left\|\partial_{\mathbf{y}}^{\boldsymbol{\alpha}} f_{\mathrm{ref}}\right\|_{L^{\infty}\left(\square ; L^{\infty}\left(D_{\mathrm{ref}}\right)\right)} \leq(|\boldsymbol{\alpha}|+1) ! c_{f} C_{\operatorname{det}}\left(\frac{d}{\underline{\sigma \rho} \log 2}\right)^{|\boldsymbol{\alpha}|} \boldsymbol{\gamma}^{\boldsymbol{\alpha}}
$$

Finally, we establish the dependency between the solution $\hat{u}$ to (9) and the data $f_{\text {ref }}$.

Lemma 6 Let $\hat{u}(\mathbf{y})$ be the solution to (9) and $f_{\text {ref }} \in L^{\infty}\left(\square ; L^{\infty}\left(D_{\text {ref }}\right)\right)$. Then, there holds

$$
\|\hat{u}(\mathbf{y})\|_{H^{1}\left(D_{\text {ref }}\right)} \leq \frac{\bar{\sigma}^{2}}{\underline{\sigma}^{d}} c_{D}\left\|f_{\text {ref }}\right\|_{L^{\infty}\left(\square ; L^{\infty}\left(D_{\text {ref }}\right)\right)}
$$



Proof The bilinear form

$$
(\mathbf{A} \nabla \cdot, \nabla \cdot)_{L^{2}\left(D_{\text {ref }} ; \mathbb{R}^{d}\right)}: H_{0}^{1}\left(D_{\text {ref }}\right) \times H_{0}^{1}\left(D_{\text {ref }}\right) \rightarrow \mathbb{R}
$$

is coercive and bounded according to (4) and $\underline{\sigma}^{d} \leq \operatorname{det} \mathbf{J}(\mathbf{x}, \mathbf{y}) \leq \bar{\sigma}^{d}$. It holds

$$
\frac{\underline{\sigma}^{d}}{\bar{\sigma}^{2}}\|\hat{u}\|_{H^{1}\left(D_{\text {ref }}\right)}^{2} \leq(\mathbf{A} \nabla \hat{u}, \nabla \hat{u})_{L^{2}\left(D_{\text {ref }} ; \mathbb{R}^{d}\right)}
$$

and

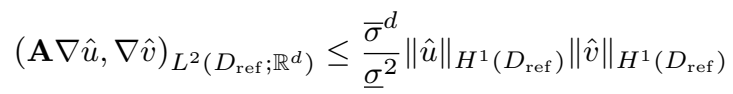

for all $\hat{u}, \hat{v} \in H^{1}\left(D_{\text {ref }}\right)$ and almost every $\mathbf{y} \in \square$. The assertion follows now by the application of the Lax-Milgram Lemma and the observation that

$$
\left\|f_{\text {ref }}\right\|_{L^{\infty}\left(\square ; H^{-1}\left(D_{\text {ref }}\right)\right)} \leq \sqrt{\left|D_{\text {ref }}\right|} c_{P}\left\|f_{\text {ref }}\right\|_{L^{\infty}\left(\square ; L^{\infty}\left(D_{\text {ref }}\right)\right)},
$$

where $c_{P}$ denotes the Poincaré constant of $D_{\text {ref }}$. 
Combining the constants arising from Theorem 3 and Theorem 4 leads to the modified sequence

$$
\left\{\mu_{k}\right\}_{k}:=\left\{2 \max \left(\frac{d}{\underline{\sigma \rho} \log 2}, \frac{2\left(1+c_{\gamma}\right)}{\underline{\sigma}^{2} \log 2}\right) \gamma_{k}\right\}_{k}
$$

such that

$$
\left\|\partial_{\mathbf{y}}^{\boldsymbol{\alpha}} \mathbf{A}\right\| \|_{d \times d} \leq C|\boldsymbol{\alpha}| ! \boldsymbol{\mu}^{\boldsymbol{\alpha}} \quad \text { and } \quad\left\|\partial_{\mathbf{y}}^{\boldsymbol{\alpha}} f_{\mathrm{ref}}\right\|_{L^{\infty}\left(\square ; L^{\infty}\left(D_{\mathrm{ref}}\right)\right)} \leq C|\boldsymbol{\alpha}| ! \boldsymbol{\mu}^{\boldsymbol{\alpha}} .
$$

Herein, we set $C:=C_{\text {det }} \max \left(c_{f}, 1 / \underline{\sigma}^{2}\right)$. Notice that we introduced also the additional factor 2 in order to obtain the factor $|\boldsymbol{\alpha}|$ ! in the derivatives instead of the factor $(|\boldsymbol{\alpha}|+1)$ !.

Theorem 5 The derivatives of the solution $u$ to (9) satisfy under the conditions of Assumption1.3 that

$$
\left\|\partial_{\mathbf{y}}^{\boldsymbol{\alpha}} \hat{u}(\mathbf{y})\right\|_{H^{1}\left(D_{\mathrm{ref}}\right)} \leq|\boldsymbol{\alpha}| ! \boldsymbol{\mu}^{\boldsymbol{\alpha}}\left(4 \frac{\bar{\sigma}^{2}}{\underline{\sigma}^{d}} C \max \left\{1, c_{D}\right\}\right)^{|\boldsymbol{\alpha}|+1},
$$

where $c_{D}$ denotes the constant from the previous theorem.

Proof Differentiating the variational formulation (9) with respect to $\mathbf{y}$ leads to

$$
\left(\partial_{\mathbf{y}}^{\boldsymbol{\alpha}}\left(\mathbf{A}(\mathbf{y}) \nabla_{\mathbf{x}} \hat{u}(\mathbf{y})\right), \nabla_{\mathbf{x}} \hat{v}\right)_{L^{2}\left(D_{\mathrm{ref}} ; \mathbb{R}^{d}\right)}=\left(\partial_{\mathbf{y}}^{\boldsymbol{\alpha}} f_{\mathrm{ref}}(\mathbf{y}), \hat{v}\right)_{L^{2}\left(D_{\mathrm{ref}} ; \mathbb{R}\right)} .
$$

The isomorphism of the spaces $H_{0}^{1}\left(D_{\text {ref }}\right)$ and $H_{0}^{1}(D(\mathbf{y}))$ from Lemma 1 allows us to consider the test functions $v$ to be independent of $\mathbf{y}$. Furthermore, the application of the Leibniz rule for the expression $\partial_{\mathbf{y}}^{\boldsymbol{\alpha}}\left(\mathbf{A}(\mathbf{y}) \nabla_{\mathbf{x}} \hat{u}(\mathbf{y})\right)$ results in

$$
\partial_{\mathbf{y}}^{\boldsymbol{\alpha}}\left(\mathbf{A}(\mathbf{y}) \nabla_{\mathbf{x}} \hat{u}(\mathbf{y})\right)=\sum_{\boldsymbol{\alpha}^{\prime} \leq \boldsymbol{\alpha}}\left(\begin{array}{c}
\boldsymbol{\alpha} \\
\boldsymbol{\alpha}^{\prime}
\end{array}\right) \partial_{\mathbf{y}}^{\boldsymbol{\alpha}^{\prime}} \mathbf{A}(\mathbf{y}) \partial_{\mathbf{y}}^{\boldsymbol{\alpha}-\boldsymbol{\alpha}^{\prime}} \nabla_{\mathbf{x}} \hat{u}(\mathbf{y})
$$

Thus, rearranging the preceding expression and using the linearity of the gradient, we arrive at

$$
\begin{aligned}
& \int_{D_{\mathrm{ref}}} \mathbf{A}(\mathbf{y}) \nabla_{\mathbf{x}} \partial_{\mathbf{y}}^{\boldsymbol{\alpha}} \hat{u}(\mathbf{y}) \nabla_{\mathbf{x}} v \mathrm{~d} \mathbf{x} \\
& \quad=\int_{D_{\mathrm{ref}}} \partial_{\mathbf{y}}^{\boldsymbol{\alpha}} f_{\mathrm{ref}}(\mathbf{y}) v \mathrm{~d} \mathbf{x}-\sum_{\boldsymbol{\alpha} \neq \boldsymbol{\alpha}^{\prime} \leq \boldsymbol{\alpha}}\left(\begin{array}{c}
\boldsymbol{\alpha} \\
\boldsymbol{\alpha}^{\prime}
\end{array}\right) \int_{D_{\text {ref }}} \partial_{\mathbf{y}}^{\boldsymbol{\alpha}-\boldsymbol{\alpha}^{\prime}} \mathbf{A}(\mathbf{y}) \nabla_{\mathbf{x}} \partial_{\mathbf{y}}^{\boldsymbol{\alpha}^{\prime}} \hat{u}(\mathbf{y}) \nabla_{\mathbf{x}} v \mathrm{~d} \mathbf{x} .
\end{aligned}
$$

By choosing $v=\partial_{\mathbf{y}}^{\boldsymbol{\alpha}} \hat{u}(\mathbf{y})$ and by employing the estimates from Theorem 3 and Theorem 4, it follows that

$$
\begin{aligned}
& \frac{\frac{\sigma}{d}^{d}}{\bar{\sigma}^{2}}\left\|\partial_{\mathbf{y}}^{\boldsymbol{\alpha}} \hat{u}(\mathbf{y})\right\|_{H^{1}\left(D_{\mathrm{ref}}\right)}^{2} \\
& \leq \int_{D_{\text {ref }}} \partial_{\mathbf{y}}^{\boldsymbol{\alpha}} f_{\text {ref }}(\mathbf{y}) \partial_{\mathbf{y}}^{\boldsymbol{\alpha}} \hat{u}(\mathbf{y}) \mathrm{d} \mathbf{x}-\sum_{\boldsymbol{\alpha} \neq \boldsymbol{\alpha}^{\prime} \leq \boldsymbol{\alpha}}\left(\begin{array}{c}
\boldsymbol{\alpha} \\
\boldsymbol{\alpha}^{\prime}
\end{array}\right) \int_{D_{\text {ref }}} \partial_{\mathbf{y}}^{\boldsymbol{\alpha}-\boldsymbol{\alpha}^{\prime}} \mathbf{A}(\mathbf{y}) \nabla_{\mathbf{x}} \partial_{\mathbf{y}}^{\boldsymbol{\alpha}^{\prime}} \hat{u}(\mathbf{y}) \nabla_{\mathbf{x}} \partial_{\mathbf{y}}^{\boldsymbol{\alpha}} \hat{u}(\mathbf{y}) \mathrm{d} \mathbf{x} \\
& \leq c_{D} C|\boldsymbol{\alpha}| ! \boldsymbol{\mu}^{\boldsymbol{\alpha}}\left\|\partial_{\mathbf{y}}^{\boldsymbol{\alpha}} \hat{u}(\mathbf{y})\right\|_{H^{1}\left(D_{\text {ref }}\right)} \\
& +\sum_{\boldsymbol{\alpha} \neq \boldsymbol{\alpha}^{\prime} \leq \boldsymbol{\alpha}}\left(\begin{array}{c}
\boldsymbol{\alpha} \\
\boldsymbol{\alpha}^{\prime}
\end{array}\right) C\left|\boldsymbol{\alpha}-\boldsymbol{\alpha}^{\prime}\right| ! \boldsymbol{\mu}^{\boldsymbol{\alpha}-\boldsymbol{\alpha}^{\prime}}\left\|\partial_{\mathbf{y}}^{\boldsymbol{\alpha}^{\prime}} \hat{u}(\mathbf{y})\right\|_{H^{1}\left(D_{\mathrm{ref}}\right)}\left\|\partial_{\mathbf{y}}^{\boldsymbol{\alpha}} \hat{u}(\mathbf{y})\right\|_{H^{1}\left(D_{\mathrm{ref}}\right)} .
\end{aligned}
$$


From this, we obtain

$$
\left\|\partial_{\mathbf{y}}^{\boldsymbol{\alpha}} \hat{u}(\mathbf{y})\right\|_{H^{1}\left(D_{\mathrm{ref}}\right)} \leq \frac{\tilde{C}}{4}|\boldsymbol{\alpha}| ! \boldsymbol{\mu}^{\boldsymbol{\alpha}}+\frac{\tilde{C}}{4} \sum_{\boldsymbol{\alpha} \neq \boldsymbol{\alpha}^{\prime} \leq \boldsymbol{\alpha}}\left(\begin{array}{c}
\boldsymbol{\alpha} \\
\boldsymbol{\alpha}^{\prime}
\end{array}\right)\left|\boldsymbol{\alpha}-\boldsymbol{\alpha}^{\prime}\right| ! \boldsymbol{\mu}^{\boldsymbol{\alpha}-\boldsymbol{\alpha}^{\prime}}\left\|\partial_{\mathbf{y}}^{\boldsymbol{\alpha}^{\prime}} \hat{u}(\mathbf{y})\right\|_{H^{1}\left(D_{\mathrm{ref}}\right)}
$$

by setting

$$
\tilde{C}:=4 \frac{\bar{\sigma}^{2}}{{\underline{\sigma^{d}}}^{d}} C \max \left(1, c_{D}\right)
$$

The proof is now by induction on $|\boldsymbol{\alpha}|$. The induction hypothesis is given by

$$
\left\|\partial_{\mathbf{y}}^{\boldsymbol{\alpha}} \hat{u}(\mathbf{y})\right\|_{H^{1}\left(D_{\text {ref }}\right)} \leq|\boldsymbol{\alpha}| ! \boldsymbol{\mu}^{\boldsymbol{\alpha}} \tilde{C}^{|\boldsymbol{\alpha}|+1} .
$$

For $|\boldsymbol{\alpha}|=0$, we conclude just the stability estimate (26), where the right hand side of the inequality is scaled by the factor 4 . Therefore, let the assertion hold for all $|\boldsymbol{\alpha}| \leq n-1$ for some $n \geq 1$. Then, we have

$$
\begin{aligned}
& \left\|\partial_{\mathbf{y}}^{\boldsymbol{\alpha}} \hat{u}(\mathbf{y})\right\|_{H^{1}\left(D_{\mathrm{ref}}\right)} \\
& \quad \leq \frac{\tilde{C}}{4}|\boldsymbol{\alpha}| ! \boldsymbol{\mu}^{\boldsymbol{\alpha}}+\frac{\tilde{C}}{4} \sum_{\boldsymbol{\alpha \neq \boldsymbol { \alpha } ^ { \prime } \leq \boldsymbol { \alpha }}}\left(\begin{array}{c}
\boldsymbol{\alpha} \\
\boldsymbol{\alpha}^{\prime}
\end{array}\right)\left|\boldsymbol{\alpha}-\boldsymbol{\alpha}^{\prime}\right| ! \boldsymbol{\mu}^{\boldsymbol{\alpha}-\boldsymbol{\alpha}^{\prime}}\left|\boldsymbol{\alpha}^{\prime}\right| ! \boldsymbol{\mu}^{\boldsymbol{\alpha}^{\prime}} \tilde{C}^{\left|\boldsymbol{\alpha}^{\prime}\right|+1} \\
& \quad \leq \frac{\tilde{C}}{4}|\boldsymbol{\alpha}| ! \boldsymbol{\mu}^{\boldsymbol{\alpha}}+\frac{\tilde{C}}{4} \boldsymbol{\mu}^{\boldsymbol{\alpha}} \sum_{\boldsymbol{\alpha} \neq \boldsymbol{\alpha}^{\prime} \leq \boldsymbol{\alpha}}\left(\begin{array}{c}
\boldsymbol{\alpha} \\
\boldsymbol{\alpha}^{\prime}
\end{array}\right)\left|\boldsymbol{\alpha}-\boldsymbol{\alpha}^{\prime}\right| ! \tilde{C}^{\left|\boldsymbol{\alpha}^{\prime}\right|+1} \\
& \quad=\frac{\tilde{C}}{4}|\boldsymbol{\alpha}| ! \boldsymbol{\mu}^{\boldsymbol{\alpha}}+\frac{\tilde{C}}{4} \boldsymbol{\mu}^{\boldsymbol{\alpha}} \sum_{j=0}^{n-1} \sum_{\substack{\boldsymbol{\alpha}^{\prime} \leq \boldsymbol{\alpha} \\
\left|\boldsymbol{\alpha}^{\prime}\right|=j}}\left(\begin{array}{c}
\boldsymbol{\alpha} \\
\boldsymbol{\alpha}^{\prime}
\end{array}\right)\left|\boldsymbol{\alpha}-\boldsymbol{\alpha}^{\prime}\right| !\left|\boldsymbol{\alpha}^{\prime}\right| ! \tilde{C}^{\left|\boldsymbol{\alpha}^{\prime}\right|+1}
\end{aligned}
$$

Again, we make use of the combinatorial identity (25) and obtain the estimate

$$
\begin{aligned}
\left\|\partial_{\mathbf{y}}^{\boldsymbol{\alpha}} \hat{u}(\mathbf{y})\right\|_{H^{1}\left(D_{\mathrm{ref}}\right)} & \leq \frac{\tilde{C}}{4}|\boldsymbol{\alpha}| ! \boldsymbol{\mu}^{\boldsymbol{\alpha}}+\frac{\tilde{C}}{4}|\boldsymbol{\alpha}| ! \boldsymbol{\mu}^{\boldsymbol{\alpha}} \sum_{j=0}^{n-1}\left(\begin{array}{c}
|\boldsymbol{\alpha}| \\
j
\end{array}\right)(|\boldsymbol{\alpha}|-j) ! j ! \tilde{C}^{j+1} \\
& =\frac{\tilde{C}}{4}|\boldsymbol{\alpha}| ! \boldsymbol{\mu}^{\boldsymbol{\alpha}}+\frac{\tilde{C}}{4}|\boldsymbol{\alpha}| ! \boldsymbol{\mu}^{\boldsymbol{\alpha}} \tilde{C} \sum_{j=0}^{n-1} \tilde{C}^{j} \\
& \leq \frac{\tilde{C}}{4}|\boldsymbol{\alpha}| ! \boldsymbol{\mu}^{\boldsymbol{\alpha}}+\frac{\tilde{C}}{4}|\boldsymbol{\alpha}| ! \boldsymbol{\mu}^{\boldsymbol{\alpha}} \tilde{C} \frac{\tilde{C}^{|\boldsymbol{\alpha}|}}{\tilde{C}-1}
\end{aligned}
$$

Now, the application of Lemma 9 from the Appendix gives us

$$
\frac{\tilde{C}}{2} \frac{\tilde{C}^{|\boldsymbol{\alpha}|}}{\tilde{C}-1} \leq \tilde{C}^{|\boldsymbol{\alpha}|}
$$

Since $\tilde{C}>1$, we conclude

$$
\left\|\partial_{\mathbf{y}}^{\boldsymbol{\alpha}} \hat{u}(\mathbf{y})\right\|_{H^{1}\left(D_{\mathrm{ref}}\right)} \leq \frac{\tilde{C}^{|\boldsymbol{\alpha}|+1}}{4}|\boldsymbol{\alpha}| ! \boldsymbol{\mu}^{\boldsymbol{\alpha}}+\frac{\tilde{C}^{|\boldsymbol{\alpha}|+1}}{2}|\boldsymbol{\alpha}| ! \boldsymbol{\mu}^{\boldsymbol{\alpha}} \leq \tilde{C}^{|\boldsymbol{\alpha}|+1}|\boldsymbol{\alpha}| ! \boldsymbol{\mu}^{\boldsymbol{\alpha}} .
$$

This completes the proof. 
Taking into account the additional factor provided by the theorem, we end up with the sequence

$$
\left\{\mu_{k}\right\}_{k}:=\left\{\frac{8 \bar{\sigma}^{2}}{\underline{\sigma}^{d}} C \max \left(1, c_{D}\right) \max \left(\frac{d}{\underline{\sigma} \rho \log 2}, \frac{2\left(1+c_{\gamma}\right)}{\underline{\sigma}^{2} \log 2}\right) \gamma_{k}\right\}_{k},
$$

which yields in view of Theorem 5 that

$$
\left\|\partial_{\mathbf{y}}^{\boldsymbol{\alpha}} \hat{u}(\mathbf{y})\right\|_{H^{1}\left(D_{\mathrm{ref}}\right)} \leq C|\boldsymbol{\alpha}| ! \boldsymbol{\mu}^{\boldsymbol{\alpha}}
$$

with a constant $C>0$ independent of the dimension $M$. Moreover, we observe $\mu_{k} \approx \gamma_{k}$. Therefore, we obtain for $\gamma_{k} \lesssim k^{-1-\delta}$ the analyticity of $\hat{u}$ by Lemma 8 from the Appendix for any $\delta>0$.

Remark 2 The discussion in this section only refers to the case of the Poisson equation. Of course, the analysis presented here straightforwardly applies also to the more general diffusion problem

$$
-\operatorname{div}(\alpha(\mathbf{x}) \nabla u(\mathbf{x}, \mathbf{y}))=f(\mathbf{x}) \quad \text { for } \mathbf{x} \in D(\mathbf{y}) .
$$

In this case, one has to impose the restriction that $\alpha(\mathbf{x})$ is an analytic function which is bounded from above and below away from 0 . Then, an estimate analogous to Lemma 5 applies for $\hat{\alpha}(\mathbf{x}, \mathbf{y})$. The proof of a related Theorem 3 for $\hat{\alpha}(\mathbf{x}, \mathbf{y}) \mathbf{A}(\mathbf{x}, \mathbf{y})$ then involves an additional application of the Leibniz rule.

Remark 3 We can obtain similar approximation results for the moments of $\hat{u}$, i.e. for $\hat{u}^{p}$ with $p \in \mathbb{N}$, possibly with worse constants. To that end, one has to bound the derivatives of $\hat{u}^{p}$ with respect to $\mathbf{y}$, too. This is also achieved by the application of Faà di Bruno's formula. For an idea of the related proofs, we refer to [16] where this topic is discussed in case of a random diffusion coefficient.

\section{Curved domains and parametric finite elements}

For the analysis of the regularity in the preceding section, we have exploited that there exists a one-to-one correspondence between the deterministic problem on the random domain and the random problem on the reference domain. For the computations, in contrast to $[6,36]$, we do however not aim at mapping the equation to the reference domain $D_{\text {ref }}$ but rather to solve the equation on each particular realization $D\left(\mathbf{y}_{i}\right)=\mathbf{V}\left(D_{\text {ref }}, \mathbf{y}_{i}\right)$ for a suitable set of samples $\left\{\mathbf{y}_{i}\right\}_{i=1}^{N} \subset \square$. A first step towards this approach is made by [26], where a random boundary variation is assumed and a mesh on the realization $D\left(\mathbf{y}_{i}\right)$ is generated via the solution of the Laplacian. Here, under the assumption that the random domain is obtained by a sufficiently smooth mapping $\mathbf{V}\left(\mathbf{y}_{i}\right)$, we will employ parametric finite elements to map the mesh on $D_{\text {ref }}$ onto a mesh on $D\left(\mathbf{y}_{i}\right)$.

We assume that the domain $D_{\text {ref }}$ is given as a collection of simplicial smooth patches. More precisely, let $\triangle$ denote the reference simplex in $\mathbb{R}^{d}$. We assume that the domain $D_{\text {ref }}$ is partitioned into $K$ patches

$$
\overline{D_{\text {ref }}}=\bigcup_{j=1}^{K} \tau_{0, j}, \quad \tau_{0, j}=\boldsymbol{\kappa}_{j}(\triangle), \quad j=1,2, \ldots, K,
$$




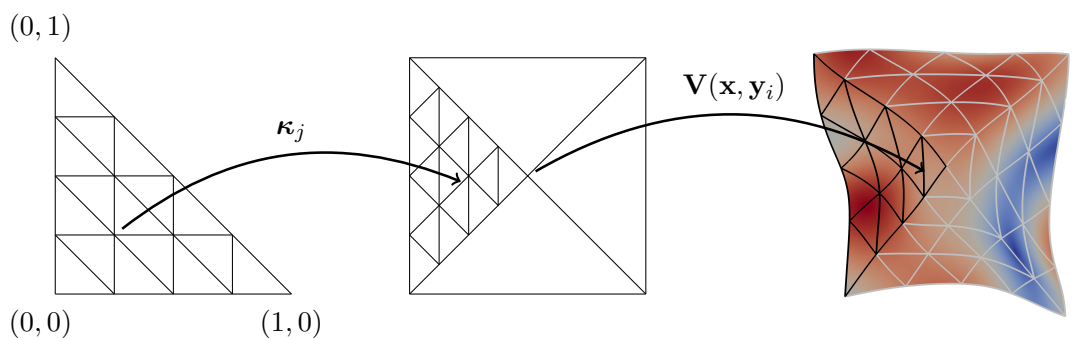

Fig. 1 Construction of parametric finite elements.

where each $\boldsymbol{\kappa}_{j}: \triangle \rightarrow \tau_{0, j}$ defines a diffeomorphism of $\triangle$ onto $\tau_{0, j}$. Thus, we have especially that

$$
\frac{\sup \left\{\left\|\boldsymbol{\kappa}_{j}^{\prime}(\mathbf{s}) \mathbf{x}\right\|_{2}: \mathbf{s} \in \triangle,\|\mathbf{x}\|_{2}=1\right\}}{\inf \left\{\left\|\boldsymbol{\kappa}_{j}^{\prime}(\mathbf{s}) \mathbf{x}\right\|_{2}: \mathbf{s} \in \triangle,\|\mathbf{x}\|_{2}=1\right\}} \leq \rho_{j} \quad \text { for all } j=1, \ldots, K,
$$

where $\boldsymbol{\kappa}_{j}^{\prime}$ denotes as before the Jacobian of $\boldsymbol{\kappa}_{j}$. Since there are only finitely many patches, we may set $\rho:=\max _{j=1}^{K} \rho_{j}$. The intersection $\tau_{0, j} \cap \tau_{0, j^{\prime}}, j \neq j^{\prime}$, of any two patches $\tau_{0, j}$ and $\tau_{0, j^{\prime}}$ is supposed to be either $\emptyset$, or a common lower dimensional face.

A mesh on level $\ell$ on $D_{\text {ref }}$ is now obtained by regular subdivisions of depth $\ell$ of the reference simplex into $2^{\ell d}$ sub-simplices. This generates the $2^{\ell d}$ elements $\left\{\tau_{\ell, j}\right\}_{j}$. In order to ensure that the triangulation $\mathcal{T}_{\ell}:=\left\{\tau_{\ell, j}\right\}_{j}$ on the level $\ell$ forms a regular mesh on $D_{\text {ref }}$, the parametrizations $\left\{\boldsymbol{\kappa}_{j}\right\}_{j}$ are assumed to be $C^{0}$ compatible in the following sense: there exists a bijective, affine mapping $\boldsymbol{\Xi}: \triangle \rightarrow \triangle$ such that for all $\mathbf{x}=\boldsymbol{\kappa}_{i}(\mathbf{s})$ on a common interface of $\tau_{0, j}$ and $\tau_{0, j^{\prime}}$ it holds that $\boldsymbol{\kappa}_{j}(\mathbf{s})=\left(\boldsymbol{\kappa}_{j^{\prime}}\right.$ ○ $\boldsymbol{\Xi})(\mathbf{s})$. In other words, the diffeomorphisms $\boldsymbol{\kappa}_{j}$ and $\boldsymbol{\kappa}_{j^{\prime}}$ coincide at the common interface except for orientation. An illustration of such a triangulation is found in Figure 1. Notice that in our construction the local element mappings $\Delta \rightarrow \tau_{\ell, j}$ satisfy the same bound (28) by definition. Therefore, especially the uniformity condition for (iso-) parametric finite elements is fulfilled, cf. [4,22].

Finally, we define the finite element ansatz functions via the parametrizations $\left\{\boldsymbol{\kappa}_{j}\right\}_{j}$ in the usual fashion, i.e. by lifting Lagrangian finite elements from $\triangle$ to the domain $D_{\text {ref }}$ by using the mappings $\boldsymbol{\kappa}_{j}$. To that end, we define on the $\ell$-th subdivision $\triangle_{\ell}$ of the reference domain the standard Lagrangian piecewise polynomial continuous finite elements $\Phi_{\ell}=\left\{\varphi_{\ell, i}: i \in \mathcal{I}_{\ell}\right\}$, where $\mathcal{I}_{\ell}$ denotes an appropriate index set. The corresponding finite element space is then given by

$$
V_{\triangle, \ell}=\operatorname{span}\left\{\varphi_{\ell, j}: j \in \mathcal{I}_{\ell}\right\}=\left\{u \in C(\triangle):\left.u\right|_{\tau} \in \Pi_{n} \text { for all } \tau \in \triangle_{\ell}\right\}
$$

with $\operatorname{dim} V_{\triangle, \ell} \approx 2^{\ell d}$ and $\Pi_{n}$ denoting the space of polynomials of degree at most $n$. Continuous basis functions whose support overlaps with several patches are obtained by gluing across patch boundaries, using the $C^{0}$ inter-patch compatibility. This yields a (nested) sequence of finite element spaces

$$
V_{\text {ref }, \ell}:=\left\{v \in C\left(D_{\text {ref }}\right):\left.v\right|_{\boldsymbol{\kappa}_{j}(\triangle)}=\varphi \circ \boldsymbol{\kappa}_{j}^{-1}, \varphi \in V_{\triangle, \ell}, j=1, \ldots, K\right\} \subset H^{1}\left(D_{\text {ref }}\right)
$$


with $\operatorname{dim} V_{\text {ref, } \ell} \approx 2^{\ell d}$. It is well known that the spaces $V_{\text {ref, } \ell}$ satisfy the following Jackson and Bernstein type estimates for all $0 \leq s \leq t<3 / 2, t \leq q \leq n+1$

$$
\inf _{v_{\ell} \in V_{\mathrm{ref}, \ell}}\left\|u-v_{\ell}\right\|_{H^{t}\left(D_{\mathrm{ref}}\right)} \lesssim h_{\ell}^{q-t}\|u\|_{H^{q}\left(D_{\mathrm{ref}}\right)}, \quad u \in H^{q}\left(D_{\mathrm{ref}}\right),
$$

and

$$
\left\|v_{\ell}\right\|_{H^{t}\left(D_{\text {ref }}\right)} \lesssim h_{\ell}^{s-t}\left\|v_{\ell}\right\|_{H^{s}\left(D_{\text {ref }}\right)}, \quad v_{\ell} \in V_{\text {ref }, \ell}
$$

uniformly in $\ell$, where we set $h_{\ell}:=2^{-\ell}$. Note that, by construction, $h_{\ell}$ scales like the mesh size $\max _{k}\left\{\operatorname{diam} \tau_{\ell, k}\right\}$, i.e. it holds $h_{\ell} \approx \max _{k}\left\{\operatorname{diam} \tau_{\ell, k}\right\}$ uniformly in $\ell \in \mathbb{N}$ due to $(28)$.

We can employ the same argumentation to map the finite elements from the reference domain $D_{\text {ref }}$ to the particular realization $D(\mathbf{y})=\mathbf{V}\left(D_{\text {ref }}, \mathbf{y}\right)$ for $\mathbf{y} \in \square$. The ellipticity condition (4) on the Jacobian $\mathbf{J}(\mathbf{x}, \omega)$ of the random vector field guarantees that (28) is satisfied with $\rho=\bar{\sigma} / \underline{\sigma}$. Also the Jackson and Bernstein type estimates (29) and (30) are still valid, where the only limitation is imposed by the smoothness of $\mathbf{V}(\mathbf{x}, \mathbf{y})$. If for example $\mathbf{V}(\mathbf{x}, \mathbf{y})$ is of class $C^{2}$, then we have the restriction $q \leq 2$ such that

$$
\inf _{v_{\ell} \in V_{\ell}(\mathbf{y})}\left\|u-v_{\ell}\right\|_{H^{t}(D(\mathbf{y}))} \lesssim h_{\ell}^{q-t}\|u\|_{H^{q}(D(\mathbf{y}))}
$$

for all $0 \leq t \leq 3 / 2, t \leq q \leq 2$ where $V_{\ell}(\mathbf{y}):=\left\{\varphi \circ \mathbf{V}(\mathbf{y})^{-1}: \varphi \in V_{\text {ref }, \ell}\right\} \subset H^{1}(D(\mathbf{y}))$.

The one-to-one correspondence between the solution $u_{\ell}(\mathbf{y}) \in V_{\ell}(\mathbf{y})$ to $(6)$ and the solution $\hat{u}_{\ell}(\mathbf{y}) \in V_{\text {ref }, \ell}$ to $(9)$ is given by the following

Theorem 6 Let $u_{\ell}(\mathbf{y}) \in V_{\ell}(\mathbf{y})$ be the Galerkin solution to $(6)$ and $\hat{u}_{\ell}(\mathbf{y}) \in V_{\text {ref, },}$ the Galerkin solution to (9), respectively. Then, it holds

$$
\hat{u}_{\ell}(\mathbf{y})=u_{\ell} \circ \mathbf{V}(\mathbf{y}) \quad \text { and } \quad u_{\ell}(\mathbf{y})=\hat{u}_{\ell} \circ \mathbf{V}(\mathbf{y})^{-1} .
$$

Proof The proof is a straightforward consequence of the construction of the spaces $V_{\ell}(\mathbf{y})$ and the equivalence of the problems (6) and (9), see also (10).

Remark 4 The $H^{2}$-regularity of the mapped problem, i.e. on $D(\mathbf{y})$, follows from the $H^{2}$-regularity of the problem on the reference domain $D_{\text {ref }}$ if the vector field $\mathbf{V}(\mathbf{x}, \mathbf{y})$ is at least a $C^{2}$-diffeomorphism. Especially, if $\mathbf{V}(\mathbf{x}, \mathbf{y})=\mathbf{x}+\mathbf{V}_{0}(\mathbf{x}, \mathbf{y})$ is a perturbation of the identity as in (16) and $\mathbf{V}_{0}(\mathbf{x}, \mathbf{y})$ is of class $C^{2}$, then $\mathbf{V}(\mathbf{x}, \mathbf{y})^{-1}$ is also a $C^{2}$-diffeomorphism provided that $\left\|\mathbf{V}_{0}(\cdot, \mathbf{y})\right\|_{C^{2}\left(D_{\text {ref }}\right)}<1 / 2$, cf. [31].

\section{Stochastic interface problems}

As a special case of a diffusion problem on a random domain, we shall focus on the stochastic interface problem as already discussed in e.g. [14]. 


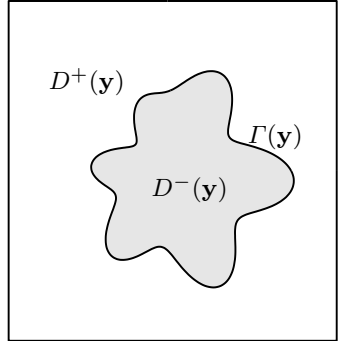

Fig. 2 Visualization of the domain $D$ and the random interface $\Gamma(\mathbf{y})$.

\subsection{Problem formulation}

Let the hold-all $\mathcal{D} \subset \mathbb{R}^{d}$, cf. (3), be a simply-connected and convex domain with Lipschitz continuous boundary $\partial \mathcal{D}$. Inscribed into $\mathcal{D}$, we have a randomly varying inclusion $D^{-}(\mathbf{y}) \subsetneq \mathcal{D}$ for $\mathbf{y} \in \square$ with a $C^{2}$-smooth boundary $\Gamma(\mathbf{y}):=\partial D^{-}(\mathbf{y})$. The complement of $\overline{D^{-}(\mathbf{y})}$ will be denoted by $D^{+}(\mathbf{y}):=\mathcal{D} \backslash \overline{D^{-}(\mathbf{y})}$. A visualization of this setup is found in Figure 2. For given $\mathbf{y} \in \square$, we can state the stochastic elliptic interface problem as follows:

$$
\begin{aligned}
-\operatorname{div}(\alpha(\mathbf{x}, \mathbf{y}) \nabla u(\mathbf{x}, \mathbf{y})) & =f(\mathbf{x}) & & \text { in } \mathcal{D} \backslash \Gamma(\mathbf{y}), \\
\llbracket u(\mathbf{x}, \mathbf{y}) \rrbracket & =0 & & \text { on } \Gamma(\mathbf{y}), \\
\llbracket \alpha(\mathbf{x}, \mathbf{y}) \frac{\partial u}{\partial \mathbf{n}}(\mathbf{x}, \mathbf{y}) \rrbracket & =0 & & \text { on } \Gamma(\mathbf{y}), \\
u(\mathbf{x}, \mathbf{y}) & =0 & & \text { on } \partial \mathcal{D} .
\end{aligned}
$$

Here, $\mathbf{n}$ denotes the outward normal vector on $\Gamma(\mathbf{y})$. Furthermore, the diffusion coefficient is given by

$$
\alpha(\mathbf{x}, \mathbf{y}):=\chi_{D^{+}(\mathbf{y})}(\mathbf{x}) \alpha^{+}(\mathbf{x})+\chi_{D^{-}(\mathbf{y})}(\mathbf{x}) \alpha^{-}(\mathbf{x}) \quad \text { for } \mathbf{x} \in \mathcal{D},
$$

where $\chi_{D^{-}(\mathbf{y})}$ is the characteristic function of $D^{-}(\mathbf{y})$ and $\alpha^{+}, \alpha^{-}$are smooth deterministic functions with

$$
0<\underline{\alpha} \leq \alpha^{-}(\mathbf{x}), \alpha^{+}(\mathbf{x}) \leq \bar{\alpha}<\infty \quad \text { for almost every } \mathbf{x} \in \mathcal{D} .
$$

By $\llbracket u(\mathbf{x}, \mathbf{y}) \rrbracket:=u^{+}(\mathbf{x}, \mathbf{y})-u^{-}(\mathbf{x}, \mathbf{y})$, we denote the jump of the solution $u$ across $\Gamma(\mathbf{y})$, where $u^{-}(\mathbf{x}, \mathbf{y}):=\left.u\right|_{D^{-}(\mathbf{y})}$ and $u^{+}(\mathbf{x}, \mathbf{y}):=\left.u\right|_{D^{+}(\mathbf{y})}$, respectively. Analogously, we define the jump of the co-normal derivative across $\Gamma(\mathbf{y})$ via

$$
\llbracket \alpha(\mathbf{x}, \mathbf{y}) \frac{\partial u}{\partial \mathbf{n}}(\mathbf{x}, \mathbf{y}) \rrbracket:=\alpha^{+}(\mathbf{x}) \frac{\partial u}{\partial \mathbf{n}}(\mathbf{x}, \mathbf{y})-\alpha^{-}(\mathbf{x}) \frac{\partial u}{\partial \mathbf{n}}(\mathbf{x}, \mathbf{y}) .
$$

Remark 5 This formulation of the stochastic interface problem also covers the case of elliptic equations on random domains. For example, for $\alpha^{+}(\mathbf{x}) \equiv 0$ and $\alpha^{-}(\mathbf{x}) \equiv$ 1 (perfect insulation), we have the Poisson equation on $D^{-}(\mathbf{y})$ with homogeneous Neumann data on $\Gamma(\mathbf{y})$ while, for $\alpha^{+}(\mathbf{x}) \equiv \infty$ and $\alpha^{-}(\mathbf{x}) \equiv 1$ (perfect conduction), we have the Poisson equation on $D^{-}(\mathbf{y})$ with homogeneous Dirichlet data on $\Gamma(\mathbf{y})$. 
6.2 Modeling the stochastic interface

Instead of solving the stochastic interface problem by the perturbation method by means of shape sensitivity analysis as in [14,18], we propose here to apply the domain mapping approach. To that end, let $\Gamma_{\text {ref }} \subset \mathcal{D}$ denote a reference interface of class $C^{2}$ and co-dimension 1 which separates the interior domain $D_{\text {ref }}^{-}$and the outer domain $D_{\text {ref }}^{+}$. We assume that $\Gamma(\mathbf{y})$ is prescribed by the application of a vector field $\mathbf{V}: \mathcal{D} \times \square \rightarrow \mathcal{D}$, i.e. $\Gamma(\mathbf{y})=\mathbf{V}\left(\Gamma_{\text {ref }}, \mathbf{y}\right)$, which is a uniform $C^{2}$-diffeomorphism in the sense of Section 2. Furthermore, let the Jacobian of $\mathbf{V}$ satisfy the ellipticity condition (4).

As an example, we can consider here an extension of the vector field in [14], which only prescribes the perturbation at the boundary: If $\Gamma_{\text {ref }}$ is of class $C^{3}$, then its outward normal $\mathbf{n}$ is of class $C^{2}$. Thus, given a random field $\kappa: \Gamma_{\text {ref }} \times \square \rightarrow \mathbb{R}$ which satisfies $|\kappa(\mathbf{x}, \mathbf{y})| \leq \bar{\kappa}<1$ almost surely, we can define $\mathbf{V}(\mathbf{x}, \mathbf{y}):=\mathbf{x}+$ $\kappa(\mathbf{x}, \mathbf{y}) \mathbf{n}(\mathbf{x})$ for $\mathbf{x} \in \Gamma_{\text {ref }}$. A suitable extension of this vector field to the whole domain $\mathcal{D}$ is given by $\mathbf{V}(\mathbf{x}, \mathbf{y}):=\mathbf{x}+\kappa(P \mathbf{x}, \mathbf{y}) \mathbf{n}(P \mathbf{x}) B\left(\|\mathbf{x}-P \mathbf{x}\|_{2}\right)$, where $P \mathbf{x}$ is the orthogonal projection of $\mathbf{x}$ onto $\Gamma_{\text {ref }}$ and $B:[0, \infty) \rightarrow[0,1]$ is a smooth blending function with $B(0)=1$ and $B(t)=0$ for all $t \geq c$ for some constant $c \in(0, \infty)$. Notice that, if $\Gamma_{\text {ref }}$ is of class $C^{3}$, the orthogonal projection $P$ onto $\Gamma_{\text {ref }}$ and thus $\mathbf{V}(\mathbf{x}, \mathbf{y})$ is at least of class $C^{2}$, cf. [19].

6.3 Reformulation for the reference interface

For $\mathbf{y} \in \square$, the variational formulation of the interface problem (31)-(34) is given as follows: Find $u \in H_{0}^{1}(\mathcal{D})$ such that

$$
\int_{D^{-}(\mathbf{y}) \cup D^{+}(\mathbf{y})} \alpha\langle\nabla u, \nabla v\rangle \mathrm{d} \mathbf{x}=\int_{\mathcal{D}} f v \mathrm{~d} \mathbf{x} \quad \text { for all } v \in H_{0}^{1}(\mathcal{D}) .
$$

As in Section 2, we can reformulate this variational formulation relative to the reference interface. As we have for the transported coefficient

$$
\begin{aligned}
\hat{\alpha}(\mathbf{x}, \mathbf{y}) & =\chi_{\mathbf{V}\left(D_{\text {ref }}^{+}, \mathbf{y}\right)}(\mathbf{V}(\mathbf{x}, \mathbf{y})) \hat{\alpha}^{+}(\mathbf{x}, \mathbf{y})+\chi_{\mathbf{V}\left(D_{\text {ref }}^{-}, \mathbf{y}\right)}(\mathbf{V}(\mathbf{x}, \mathbf{y})) \hat{\alpha}^{-}(\mathbf{x}, \mathbf{y}) \\
& =\chi_{D_{\text {ref }}^{+}(\mathbf{x}) \hat{\alpha}^{+}}(\mathbf{x}, \mathbf{y})+\chi_{D_{\text {ref }}^{-}}(\mathbf{x}) \hat{\alpha}^{-}(\mathbf{x}, \mathbf{y}),
\end{aligned}
$$

we obtain the following variational formulation with the definition (7) of the diffusion matrix $\mathbf{A}(\mathbf{x}, \mathbf{y})$ : Find $\hat{u}(\mathbf{y}) \in H_{0}^{1}(\mathcal{D})$ such that

$$
\int_{D_{\text {ref }}^{-} \cup D_{\text {ref }}^{+}} \hat{\alpha}(\mathbf{y})\langle\mathbf{A}(\mathbf{y}) \nabla \hat{u}(\mathbf{y}), \nabla v\rangle \mathrm{d} \mathbf{x}=\int_{\mathcal{D}} \hat{f}(\mathbf{y}) v \operatorname{det} \mathbf{J}(\mathbf{y}) \mathrm{d} \mathbf{x}
$$

for all $v \in H_{0}^{1}(\mathcal{D})$. Since $\hat{\alpha}(\mathbf{x}, \mathbf{y})$ is a smooth function with respect to $\mathbf{y}$, the regularity results from Section 4 remain valid here. 
6.4 Finite element approximation for the stochastic interface problem

The application of parametric finite elements yields especially an interface-resolved triangulation for the discretization of the stochastic interface problem (31)-(34). By "interface-resolved" we mean that the vertices of elements around the interface lie exactly on the interface, cf. [7,23]. Thus, the approximation error for a particular realization $u\left(\mathbf{y}_{i}\right)$ of the solution $u(\mathbf{y})$ to the stochastic interface problem (31)-(34) can be quantified by the following theorem adopted from [23, Theorem 4.1].

Theorem $\mathbf{7}$ For $\mathbf{y} \in \square$, let $\left\{\mathcal{T}_{\ell}\right\}_{\ell>0}$ be a family of interface resolved triangulations for $\mathbf{V}(\mathcal{D}, \mathbf{y})$ and $\left\{V_{\ell}(\mathbf{y})\right\}_{\ell>0}$ the associated finite element spaces. Let $u_{\ell}(\mathbf{y})$ be the finite element solution corresponding to the realization $u(\mathbf{y})$ of the solution to the elliptic problem (31)-(34). Then, for $s=0,1$, there holds that

$$
\left\|u(\mathbf{y})-u_{\ell}(\mathbf{y})\right\|_{H^{s}(\mathcal{D})} \lesssim h_{\ell}^{2-s}\|u(\mathbf{y})\|_{H^{2}\left(D^{-}(\mathbf{y})\right) \cup H^{2}\left(D^{+}(\mathbf{y})\right)}
$$

where $H^{2}\left(D^{-}(\mathbf{y})\right) \cup H^{2}\left(D^{+}(\mathbf{y})\right)$ is the broken Sobolev space equipped by the norm

$$
\|\cdot\|_{H^{2}\left(D^{-}(\mathbf{y})\right) \cup H^{2}\left(D^{+}(\mathbf{y})\right)}:=\sqrt{\|\cdot\|_{H^{2}\left(D^{-}(\mathbf{y})\right)}^{2}+\|\cdot\|_{H^{2}\left(D^{+}(\mathbf{y})\right)}^{2}} .
$$

In view of Theorem 6 , the statement of the previous theorem is also valid for the realization of the solution which is pulled back to the domain $\mathcal{D}$ relative to the reference interface $\Gamma_{\text {ref }}$.

\section{Numerical examples}

In this section, we consider two examples for boundary value problems on random domains. On the one hand, we consider a stochastic interface problem, and on the other hand, we consider the Laplace equation on a random domain. In both examples, we employ the pivoted Cholesky decomposition, cf. [15,17], in order to approximate the Karhunen-Loève expansion of $\mathbf{V}$. The spatial discretization is performed by using piecewise linear parametric finite elements on the mapped domain $\mathbf{V}\left(D_{\text {ref }}, \mathbf{y}_{i}\right)$ for each sample $\mathbf{y}_{i}$. It would of course be also possible to perform the computations on the reference domain. In this case, the diffusion matrix $\mathbf{A}$ has to be computed from Karhunen-Loève expansion of $\mathbf{V}$ for each particular sample.

For the stochastic approximation, we employ a quasi-Monte Carlo quadrature based on $N$ Halton points $\left\{\boldsymbol{\xi}_{i}\right\}_{i=1}^{N}$ mapped to the hypercube $[-1,1]^{m}$, i.e.

$$
\mathbb{E}[\hat{u}](\mathbf{x}) \approx(Q \hat{u})(\mathbf{x}):=\frac{1}{N} \sum_{i=1}^{N} \hat{u}\left(\mathbf{x}, 2 \boldsymbol{\xi}_{i}-\mathbf{1}\right) .
$$

In accordance with [16], we have the following convergence result for this quasiMonte Carlo quadrature, which is valid for the variance of $\hat{u}$ as well.

Lemma 7 The quasi-Monte Carlo quadrature with Halton points converges for the mean of the solution $\hat{u}$ to (9) independent of the stochastic dimension $M$ if $\gamma_{k} \lesssim k^{-3-\varepsilon}$. 
More precisely, for all $\delta>0$, there exists a constant such that the quasi-Monte Carlo quadrature based on $N$ satisfies

$$
\|\mathbb{E}[\hat{u}]-Q \hat{u}\|_{H^{1}\left(D_{\text {ref }}\right)} \leq C(\delta) N^{\delta-1}
$$

where $C(\delta) \rightarrow \infty$ as $\delta \rightarrow 0$.

Proof From [16,21], we know that the error of the quasi-Monte Carlo quadrature can be estimated by the weighted Koksma-Hlawka inequality, cf. [27],

$$
\begin{aligned}
& \|(\mathbb{E}-Q) \hat{u}\|_{H_{0}^{1}(D)} \\
& \quad \leq\left(\sup _{\|\boldsymbol{\alpha}\|_{\infty}=1} w_{\boldsymbol{\alpha}}^{-\frac{1}{2}} 2^{|\boldsymbol{\alpha}|} \sup _{\mathbf{y} \in[-1,1]^{M}}\left\|\partial_{\mathbf{y}}^{\boldsymbol{\alpha}} \hat{u}(\mathbf{y})\right\|_{H_{0}^{1}(D)}\right)\left(\sum_{\|\boldsymbol{\alpha}\|_{\infty}=1} w_{\boldsymbol{\alpha}}^{\frac{1}{2}} \mathcal{D}^{\star}\left(\Xi_{\boldsymbol{\alpha}}\right)\right) .
\end{aligned}
$$

Herein, we denote by $\mathcal{D}^{\star}\left(\Xi_{\boldsymbol{\alpha}}\right)$ the star-discrepancy of the set of Halton-points on $[0,1]^{M}$ which are projected onto the dimensions where $\alpha_{k}=1$. Additionally, the factor $2^{|\boldsymbol{\alpha}|}$ appears due to the transport of $\hat{u}$ to the unit cube $[0,1]^{M}$. It is shown in [34] that the second factor in (37) is bounded by

$$
\left\{\sum_{\|\boldsymbol{\alpha}\|_{\infty}=1} w_{\boldsymbol{\alpha}}^{\frac{1}{2}} \mathcal{D}^{\star}\left(\Xi_{\boldsymbol{\alpha}}\right)\right\} \leq C(\delta) N^{-1+\delta}
$$

with a constant $C(\delta)$ which is independent of $M$ if the weights $w_{\boldsymbol{\alpha}}$ are product weights, i.e. $w_{\boldsymbol{\alpha}}=\prod_{k=1}^{M} w_{k}^{\alpha_{k}}$, and satisfy

$$
\sum_{k=1}^{\infty} w_{k}^{\frac{1}{2}} k \log k<\infty
$$

In order to bound the first product in (37), we employ the estimate

$$
\left\|\partial_{\mathbf{y}}^{\boldsymbol{\alpha}} \hat{u}(\mathbf{y})\right\|_{H^{1}\left(D_{\text {ref }}\right)} \leq C|\boldsymbol{\alpha}| ! c^{|\boldsymbol{\alpha}|} \boldsymbol{\gamma}^{\boldsymbol{\alpha}} \leq C \prod_{k=1}^{M} k c \gamma_{k}
$$

from Theorem 5 and choose the weights accordingly as $w_{k}^{1 / 2}=2 c k \gamma_{k}$. Then, the condition (38) can be rewritten as

$$
\sum_{k=1}^{\infty} 2 c \gamma_{k} k^{2} \log k<\infty
$$

which is satisfied if $\gamma_{k} \lesssim k^{-3-\varepsilon}$.

All computations have been carried out on a computing server consisting of four nodes ${ }^{4}$ with up to 64 threads. 

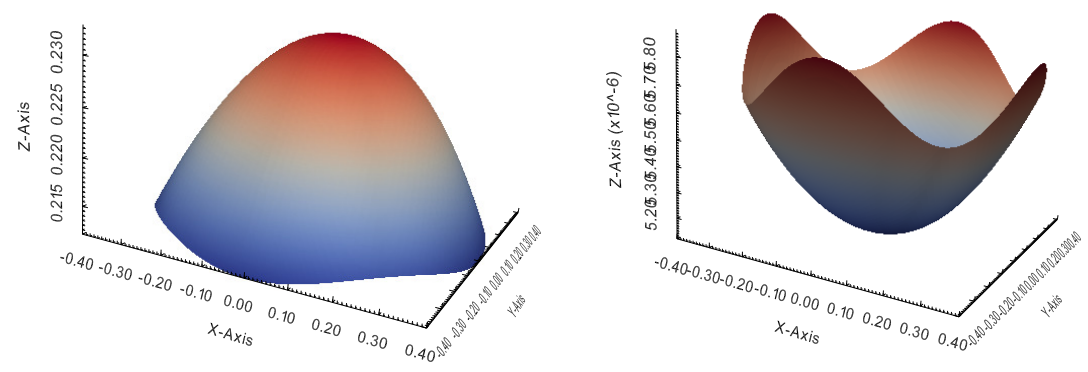

Fig. 3 Mean (left) and variance (right) of the QoI of the stochastic interface problem.

7.1 The stochastic interface problem

We consider the stochastic interface problem from [14] where the hold-all is given as $\mathcal{D}=[-1,1]^{2}$ and the reference interface is given as $\Gamma_{\text {ref }}=\left\{\mathbf{x} \in \mathcal{D}:\|\mathbf{x}\|_{2}=0.7\right\}$. Thus, the outward normal is $\mathbf{n}(\mathbf{x})=[\cos (\theta), \sin (\theta)]^{\top}$ where $\mathbf{x}=r[\cos (\theta), \sin (\theta)]^{\top}$ is the representation of $\mathbf{x}$ in polar coordinates. The random field under consideration reads

$$
\kappa(\theta, \omega)=\frac{1}{80} \sum_{k=0}^{5} \cos (k \theta) X_{2 k}(\omega)+\sin (k \theta) X_{2 k+1}(\omega) .
$$

Here, $X_{0}, \ldots, X_{11}$ are independent, uniformly distributed random variables with variance 1 , i.e. their range is $[-\sqrt{3}, \sqrt{3}]$. The diffusion coefficient is given as $\alpha^{-}(\mathbf{x}) \equiv$ 2 in the interior part of the domain and as $\alpha^{+}(\mathbf{x}) \equiv 1$ in the remaining part of the domain. The right hand side is chosen as $f(\mathbf{x}) \equiv 1$.

In this example, only the perturbation at the random interface is known. Thus, the solution of the associated diffusion problem depends on the particular extension of the vector field and it is reasonable to consider a quantity of interest (QoI) that does not depend on this extension. Specifically, the QoI is given by the solution on a non-varying part of the domain, namely on $\left\{\|\mathbf{x}\|_{2} \leq 0.4\right\}$. We therefore extend the random field (39) onto $\mathcal{D}$ as described in Subsection 6.2 by using the quadratic B-spline $B(\mathbf{x})=\frac{4}{3} B_{2}\left(5\|\mathbf{x}-P \mathbf{x}\|_{2}\right)$ as blending function. Hence, the random perturbation is localized in the annulus $\left\{0.4<\|\mathbf{x}\|_{2}<1\right\}$ and we end up with the covariance

$$
\operatorname{Cov}[\mathbf{V}](\mathbf{x}, \mathbf{y})=B(\mathbf{x}) B(\mathbf{y}) \operatorname{Cov}_{\kappa}\left(\theta_{\mathbf{x}}, \theta_{\mathbf{y}}\right)\left[\begin{array}{ll}
\cos \left(\theta_{\mathbf{x}}\right) \cos \left(\theta_{\mathbf{y}}\right) & \cos \left(\theta_{\mathbf{x}}\right) \sin \left(\theta_{\mathbf{y}}\right) \\
\sin \left(\theta_{\mathbf{x}}\right) \cos \left(\theta_{\mathbf{y}}\right) & \sin \left(\theta_{\mathbf{x}}\right) \sin \left(\theta_{\mathbf{y}}\right)
\end{array}\right]
$$

with

$$
\operatorname{Cov}_{\kappa}\left(\theta_{\mathbf{x}}, \theta_{\mathbf{y}}\right)=\frac{1}{6400} \sum_{k=0}^{5} \cos \left(k \theta_{\mathbf{x}}\right) \cos \left(k \theta_{\mathbf{y}}\right)+\sin \left(k \theta_{\mathbf{x}}\right) \sin \left(k \theta_{\mathbf{y}}\right) .
$$

Furthermore, we set $\mathbf{E}[\mathbf{V}](\mathbf{x}):=\mathbf{x}$. A visualization of the reference interface with a particular displacement field $\mathbf{V}\left(\mathbf{x}, \mathbf{y}_{i}\right)-\mathbf{x}$ and the resulting perturbed interface is found in Figure 4.

\footnotetext{
4 Each node consists of two quad-core Intel(R) Xeon(R) X5550 CPUs with a clock rate of $2.67 \mathrm{GHz}$ (hyperthreading enabled) and $48 \mathrm{~GB}$ of main memory.
} 

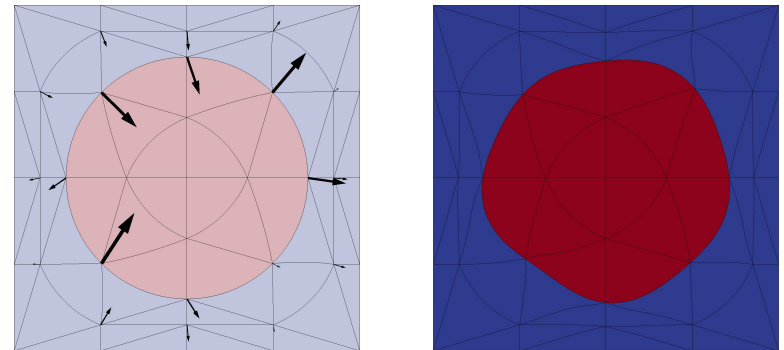

Fig. 4 Realization of the displacement $\mathbf{V}\left(\mathbf{x}, \mathbf{y}_{i}\right)-\mathbf{x}$ (left) and the related mapped interface (right).

A visualization of the QoI's mean and variance computed by $N=10^{6}$ quasiMonte Carlo samples and 1048576 finite elements (level 8) is shown in Figure 3. This approximation serves as a reference in order to examine the convergence behavior of the quasi-Monte Carlo method. According to Lemma 7, we expect a rate of convergence of $N^{\delta-1}$ for any $\delta>0$. In our experiments, we thus apply $N_{\ell}=2^{\ell /(1-\delta)}$ Halton points on the finite element level $\ell=1, \ldots, 7$ for the choices $\delta=0.5,0.4,0.3,0.2$. Although all choices of $\delta>0$ would asymptotically result in an almost linear rate of convergence, the constant in the error estimate is still dependent on the particular choice.

Figure 5 depicts the error of the solution's mean measured in the $H^{1}$-norm on the right hand side and the error of the solution's variance measured in the $W^{1,1}$-norm on the left hand side each versus the related cost, which is given by the number $N_{\ell}$ of samples times the degrees of freedom in the finite element approximation on level $\ell$. As can be seen, the error of the QoI's mean provides similar errors for all choices of $\delta$. This suggests that the finite element error limits the overall approximation error. The choice $\delta=0.2$ is already sufficient here and results in the lowest cost. For the QoI's variance, we observe successively smaller errors for increasing $\delta$. At least the error for the QoI's mean seems to be dominated by the finite element discretization. Therefore, we found it instructive to present also the respective errors measured in the $L^{2}$-norm. They are plotted in Figure 6 . Here, the smallest error is obtained for $\delta=0.5$. Nevertheless, the best error versus cost rate is provided by $\delta=0.2$. The situation changes for the variance. Here, the error gets again successively smaller for increasing values of $\delta$. Resulting in the lowest error for $\delta=0.5$.

As a comparison and in order to validate the reference, we have also computed the approximate mean and variance on each level by the Monte Carlo method. Here, in order to maintain the linear approximation rate of the finite element method in the energy norm, we approximate the root mean square error by five realizations each of which being computed with $N_{\ell}=2^{2 \ell}$ samples. 

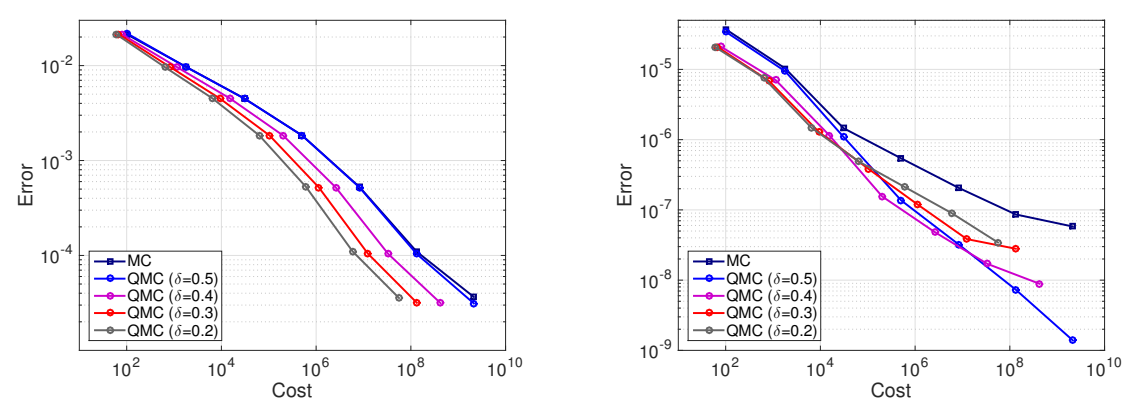

Fig. 5 Error in the mean measured in $H^{1}$ (left) and in the variance measured in $W^{1,1}$ (right).
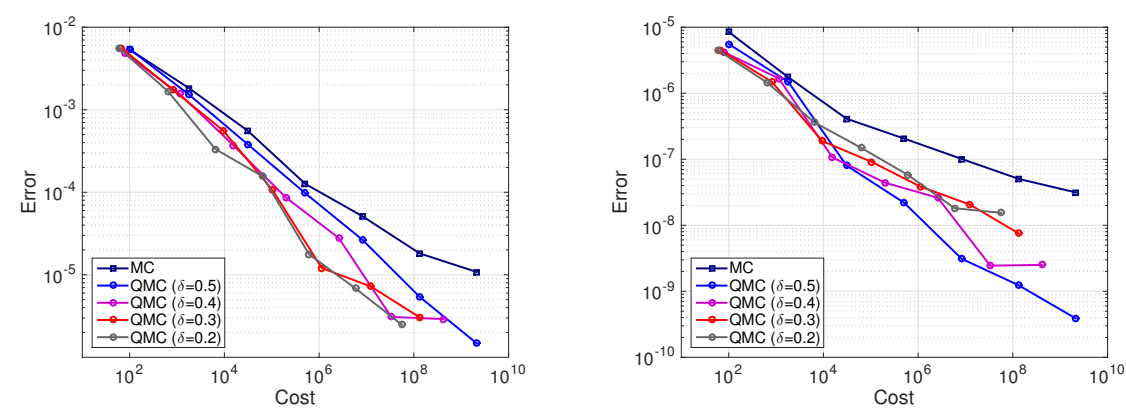

Fig. 6 Error in the mean (left) and in the variance (right) measured in $L^{2}$.
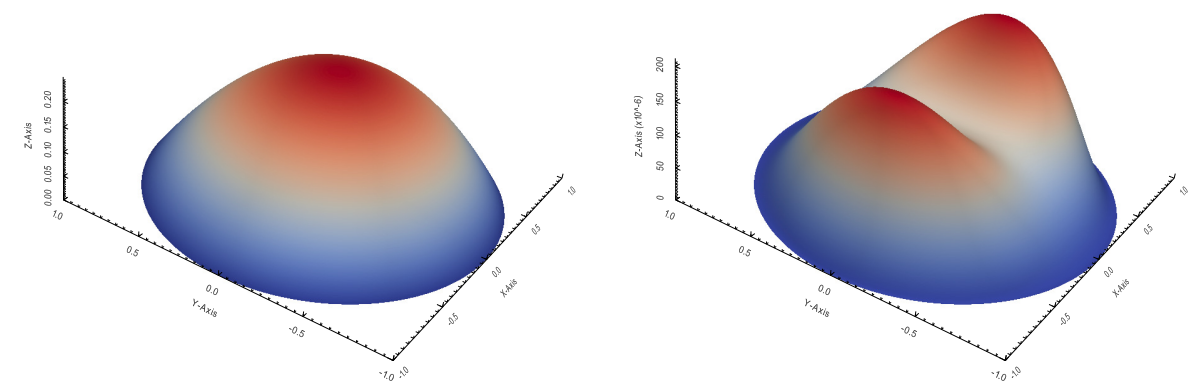

Fig. 7 Mean (left) and variance (right) of the solution $\hat{u}$ to the Laplace equation on the randomly varying disc.

7.2 The Poisson equation on a random domain

For our second example, we consider an infinite dimensional random field described by its mean $\mathbb{E}[\mathbf{V}](\mathbf{x})=\mathbf{x}$ and its covariance function

$$
\operatorname{Cov}[\mathbf{V}](\mathbf{x}, \mathbf{y})=\frac{1}{100}\left[\begin{array}{cc}
5 \exp \left(-4\|\mathbf{x}-\mathbf{y}\|_{2}^{2}\right) & \exp \left(-0.1\|2 \mathbf{x}-\mathbf{y}\|_{2}^{2}\right) \\
\exp \left(-0.1\|\mathbf{x}-2 \mathbf{y}\|_{2}^{2}\right) & 5 \exp \left(-\|\mathbf{x}-\mathbf{y}\|_{2}^{2}\right)
\end{array}\right]
$$


Furthermore, we consider the random variables in the Karhunen-Loève expansion to be uniformly distributed. The unit disc $D_{\text {ref }}=\left\{\mathbf{x} \in \mathbb{R}^{2}:\|\mathbf{x}\|_{2}<1\right\}$ serves as reference domain and the load is set to $f(\mathbf{x}) \equiv 1$. Figure 8 shows the reference domain with a particular displacement field and the resulting perturbed domain. In this example, the covariance between any two points in $D_{\text {ref }}$ is actually known and can thus be incorporated into our model. Especially, there is no point inside the reference domain that is kept fixed by the random vector field. Therefore, we consider here the entire solution $\hat{u}$ as QoI and approximate its mean and its variance.
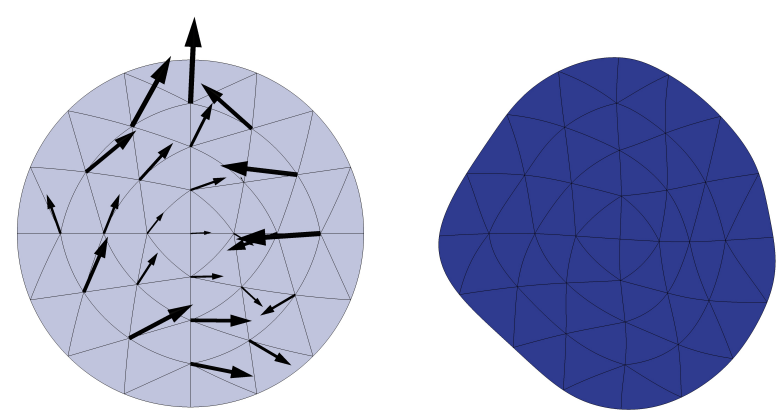

Fig. 8 Realization of the displacement $\mathbf{V}\left(\mathbf{x}, \mathbf{y}_{i}\right)-\mathbf{x}$ (left) and the related mapped domain (right).

In Figure 7, a visualization of the mean and the variance computed by $N=$ $10^{6}$ quasi-Monte Carlo samples 1048576 finite elements (level 9) are found. Here, the Karhunen-Loève expansion has been truncated after $M=303$ terms which yields a truncation error, cf. (14), smaller than $10^{-6}$. For the convergence study, however, we have coupled the truncation error of the Karhunen-Loève expansion to the spatial discretization error of order $2^{-\ell}$ on the finite element level $\ell$. It is observed that the truncation rank $M$ linearly grows in the level $\ell$, namely it holds $M=10,23,37,49,64,79,91,108$ for $\ell=1,2,3,4,5,6,7,8$.

The number of samples of the quadrature methods under consideration has been chosen in dependence on the finite element level $\ell$ as in the previous example. Figure 9 shows the error of the solution's mean and variance measured in the $H^{1}$ norm and the $W^{1,1}$-norm, respectively, each versus the cost. Except for $\delta=0.2$, we observe for the quasi-Monte Carlo quadrature as well as for the Monte Carlo quadrature comparable errors for the approximation of the mean. In view of the cost, $\delta=0.2,0.3$ perform best here. In case of the variance, we obtain again successively smaller errors for increasing values of $\delta$. Again, we have also provided the respective errors with respect to the $L^{2}$-norm. The related plots are found in Figure 10. Here, for the mean and the variance, $\delta=0.5$ provides asymptotically the lowest error. 

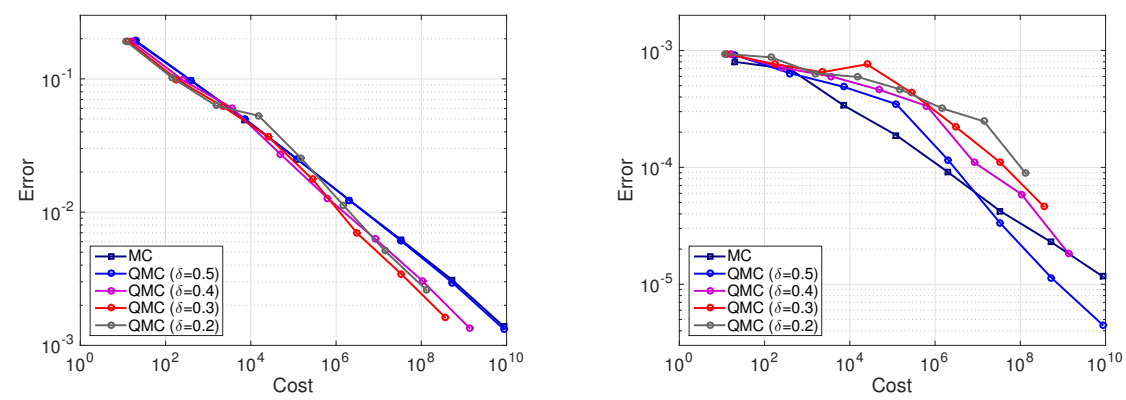

Fig. 9 Error in the mean measured in $H^{1}$ (left) and in the variance measured in $W^{1,1}$ (right).
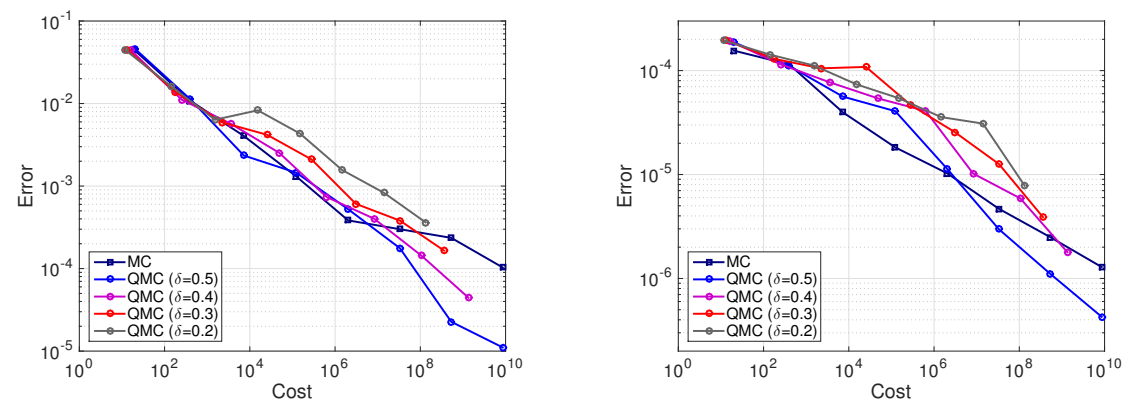

Fig. 10 Error in the mean (left) and in the variance (right) measured in $L^{2}$.

\section{Conclusion}

In this article, we have provided regularity results for the domain mapping method for elliptic boundary value problems on random domains. Based on the decay of the random vector field's Karhunen-Loève expansion, we have derived related decay rates for the solution's derivatives. In particular, the presented framework is directly applicable to stochastic interface problems. The regularity results provide dimension independent convergence of the quasi-Monte Carlo quadrature and allow also for the use of (anisotropic) quadrature methods to approximate quantities of interest that involve an integration of the solution with respect to the random parameter. The numerical examples corroborate the theoretical results and demonstrate the flexibility of the approach.

\section{Appendix}

Lemma 8 Let $\gamma=\left\{\gamma_{k}\right\}_{k} \in \ell^{1}(\mathbb{N})$ with finite support $\mathcal{I} \subset \mathbb{N}$ and $\gamma_{k} \geq 0$. Moreover, assume that $c_{\gamma}:=\sum_{k \in \mathcal{I}} \gamma_{k}<1$. Then, it holds

$$
\sum_{\alpha} \frac{|\boldsymbol{\alpha}| !}{\boldsymbol{\alpha} !} \boldsymbol{\gamma}^{\boldsymbol{\alpha}}=\frac{1}{1-c_{\gamma}}
$$

and therefore there exists a constant with $|\boldsymbol{\alpha}| ! / \boldsymbol{\alpha} ! \boldsymbol{\gamma}^{\boldsymbol{\alpha}} \leq c$ for all $\boldsymbol{\alpha} \in \mathbb{N}_{0}^{M}$, where we set $M:=|\mathcal{I}|$ and $0^{0}=1$ 
Proof It holds

$$
\sum_{\boldsymbol{\alpha}} \frac{|\boldsymbol{\alpha}| !}{\boldsymbol{\alpha} !} \boldsymbol{\gamma}^{\boldsymbol{\alpha}}=\sum_{i=0}^{\infty} \sum_{|\boldsymbol{\alpha}|=i} \frac{i !}{\boldsymbol{\alpha} !} \boldsymbol{\gamma}^{\boldsymbol{\alpha}}=\sum_{i=0}^{\infty}\left(\sum_{k=1}^{M} \gamma_{k}\right)^{i}=\sum_{i=0}^{\infty} c_{\boldsymbol{\gamma}}^{i}=\frac{1}{1-c_{\boldsymbol{\gamma}}}
$$

by the multinomial theorem and the limit of the geometric series.

Lemma 9 Let $c, m \in \mathbb{R}$ with $m \geq 2$ and $c \geq m /(m-1)$. It holds for $n \in \mathbb{N}$ that

$$
\frac{c}{m} \frac{c^{n}-1}{c-1} \leq c^{n}
$$

Proof It holds

$$
\begin{array}{rlrl} 
& & \frac{c}{m} \frac{c^{n}-1}{c-1} & \leq c^{n} \\
& \Longleftrightarrow \quad c^{n+1}-c & \leq m\left(c^{n+1}-c^{n}\right) \\
& \Longleftrightarrow \quad & m c^{n} & \leq(m-1) c^{n+1}+c \\
& \Longleftrightarrow \quad \frac{m}{m-1} & \leq c+\frac{1}{(m-1) c^{n-1}}
\end{array}
$$

Omitting the second summand together with the condition $c \geq m /(m-1)$ yields the assertion.

\section{References}

1. Abramowitz, M., Stegun, I.A.: Handbook of Mathematical Functions: With Formulas, Graphs, and Mathematical Tables. Applied mathematics series. Dover Publications, N. Chemsford, MA (1964)

2. Alt, H.W.: Lineare Funktionalanalysis. Springer, London (2007)

3. Beck, J., Tempone, R., Nobile, F., Tamellini, L.: On the optimal polynomial approximation of stochastic pdes by galerkin and collocation methods. Mathematical Models and Methods in Applied Sciences 22(9), 1250023 (2012)

4. Braess, D.: Finite Elemente: Theorie, Schnelle Löser und Anwendungen in der Elastizitätstheorie. Springer, London (2007)

5. Canuto, C., Kozubek, T.: A fictitious domain approach to the numerical solution of PDEs in stochastic domains. Numerische Mathematik 107(2), 257-293 (2007)

6. Castrillon-Candas, J.E., Nobile, F., Tempone, R.F.: Analytic regularity and collocation approximation for PDEs with random domain deformations. ArXiv e-prints 1312.7845 (2013)

7. Chen, Z., Zou, J.: Finite element methods and their convergence for elliptic and parabolic interface problems. Numerische Mathematik 79(2), 175-202 (1998)

8. Cohen, A., DeVore, R., Schwab, C.: Convergence rates of best $N$-term Galerkin approximations for a class of elliptic sPDEs. Foundations of Computational Mathematics 10, 615-646 (2010)

9. Constantine, G.M., Savits, T.H.: A multivariate Faà di Bruno formula with applications. Transactions of the American Mathematical Society 248, 503-520 (1996)

10. Ghanem, R., Spanos, P.: Stochastic finite elements: A spectral approach. Springer, New York (1991)

11. Griebel, M., Harbrecht, H.: Approximation of bi-variate functions: singular value decomposition versus sparse grids. IMA Journal of Numerical Analysis 34(1), 28-54 (2014)

12. Gross, O.A.: Preferential arrangements. American Mathematical Monthly pp. 4-8 (1962)

13. Halton, J.H.: On the efficiency of certain quasi-random sequences of points in evaluating multi-dimensional integrals. Numerische Mathematik 2(1), 84-90 (1960)

14. Harbrecht, H., Li, J.: First order second moment analysis for stochastic interface problems based on low-rank approximation. ESAIM: Mathematical Modelling and Numerical Analysis 47, 1533-1552 (2013)

15. Harbrecht, H., Peters, M., Schneider, R.: On the low-rank approximation by the pivoted Cholesky decomposition. Applied numerical mathematics 62, 28-440 (2012) 
16. Harbrecht, H., Peters, M., Siebenmorgen, M.: On the quasi-Monte Carlo method with Halton points for elliptic PDEs with log-normal diffusion. Preprint 2013-28, Mathematisches Institut Universität Basel (to appear in Mathematics of Computation) (2013)

17. Harbrecht, H., Peters, M., Siebenmorgen, M.: Efficient approximation of random fields for numerical applications. Numerical Linear Algebra with Applications 22(4), 596-617 (2015)

18. Harbrecht, H., Schneider, R., Schwab, C.: Sparse second moment analysis for elliptic problems in stochastic domains. Numerische Mathematik 109(3), 385-414 (2008)

19. Holmes, R.B.: Smoothness of certain metric projections on Hilbert space. Transactions of the American Mathematical Society 184, 87-100 (1973)

20. Kadison, R.V., Ringrose, J.R.: Fundamentals of the theory of operator algebras. V1: Elementary theory. Academic Press, New York (1986)

21. Kuo, F.Y., Schwab, C., Sloan, I.H.: Quasi-Monte Carlo methods for high-dimensional integration: the standard (weighted Hilbert space) setting and beyond. The ANZIAM Journal 53(0), 1-37 (2012)

22. Lenoir, M.: Optimal isoparametric finite elements and error estimates for domains involving curved boundaries. SIAM Journal on Numerical Analysis 23(3), 562-580 (1986)

23. Li, J., Melenk, J.M., Wohlmuth, B., Zou, J.: Optimal a priori estimates for higher order finite elements for elliptic interface problems. Applied numerical mathematics 60(1), 19-37 (2010)

24. Light, W.A., Cheney, E.W.: Approximation theory in tensor product spaces. Lecture notes in mathematics Volume 1169. Springer, New York (1985)

25. Loève, M.: Probability theory. I+II, Graduate Texts in Mathematics, vol. 45, 4th edn. Springer, New York (1977)

26. Mohan, P.S., Nair, P.B., Keane, A.J.: Stochastic projection schemes for deterministic linear elliptic partial differential equations on random domains. International Journal for Numerical Methods in Engineering 85(7), 874-895 (2011)

27. Niederreiter, H.: Random Number Generation and Quasi-Monte Carlo Methods. Society for Industrial and Applied Mathematics, Philadelphia, PA (1992)

28. Nobile, F., Tempone, R., Webster, C.G.: An anisotropic sparse grid stochastic collocation method for partial differential equations with random input data. SIAM Journal on Numerical Analysis 46(5), 2411-2442 (2008)

29. Schwab, C., Todor, R.: Karhunen-Loève approximation of random fields by generalized fast multipole methods. Journal of Computational Physics 217, 100-122 (2006)

30. Simon, B.: Methods of Modern Mathematical Physics: Functional Analysis, vol. 1. Academic Press, San Diego (1980)

31. Simon, J.: Differentiation with respect to the domain in boundary value problems. Numerical Functional Analysis and Optimization 2(7-8), 649-687 (1980)

32. Sokołowski, J., Zolésio, J.P.: Introduction to shape optimization. Shape sensitivity analysis. Springer series in computational mathematics. Springer, Berlin Heidelberg (1992)

33. Tartakovsky, D.M., Xiu, D.: Stochastic analysis of transport in tubes with rough walls. Journal of Computational Physics 217(1), 248-259 (2006)

34. Wang, X.: A constructive approach to strong tractability using quasi-Monte Carlo algorithms. Journal of Complexity 18, 683-701 (2002)

35. Wilf, H.S.: Generatingfunctionology. A. K. Peters, Ltd., Natick, MA, USA (2006)

36. Xiu, D., Tartakovsky, D.M.: Numerical methods for differential equations in random domains. SIAM Journal on Scientific Computing 28(3), 1167-1185 (2006) 